\title{
Jet quenching in hot strongly coupled gauge theories simplified
}

\section{Peter Arnold and Diana Vaman \\ Department of Physics, University of Virginia, \\ Box 400714, Charlottesville, Virginia 22904, U.S.A. \\ E-mail: parnold@virginia.edu, dv3h@virginia.edu}

ABSTRACT: Theoretical studies of jet stopping in strongly-coupled QCD-like plasmas have used gauge-gravity duality to find that the maximum stopping distance scales like $E^{1 / 3}$ for large jet energies $E$. In recent work studying jets that are created by finite-size sources in the gauge theory, we found an additional scale: the typical (as opposed to maximum) jet stopping distance scales like $(E L)^{1 / 4}$, where $L$ is the size of the space-time region where the jet is created. In this paper, we show that the results of our previous, somewhat involved computation in the gravity dual, and the $(E L)^{1 / 4}$ scale in particular, can be very easily reproduced and understood in terms of the distance that high-energy particles travel in $\mathrm{AdS}_{5}$-Schwarzschild space before falling into the black brane. We also investigate how stopping distances depend on the conformal dimension of the source operator used to create the jet.

KEYWORDS: Holography and quark-gluon plasmas, Gauge-gravity correspondence

ARXIV EPRINT: 1101.2689 


\section{Contents}

1 Introduction and results 1

2 Review of falling massless particles $\quad 6$

3 Wave packets and geometric optics $\quad 7$

3.1 Geometric optics 8

3.2 Wave packets 9

4 Reproducing the distribution of stopping distances $\quad 10$

4.1 Extracting $\mathcal{P}\left(q^{2}\right)$ from results in the literature 10

4.2 Using $\mathcal{P}\left(q^{2}\right)$ to get $\operatorname{Prob}\left(x^{3}\right) \quad 12$

5 Massive particles in $\mathbf{5}$ dimensions $\quad \mathbf{1 2}$

$\begin{array}{lll}5.1 & \text { Stopping distance of massive particles } & 13\end{array}$

$\begin{array}{lll}5.2 & \text { Generalizing the power-law tail } & 15\end{array}$

$\begin{array}{lll}5.3 & \text { Gaussian source envelope } & 17\end{array}$

6 Conclusion $\quad 18$

$\begin{array}{lr}\text { A Stopping distances in different dimensions } & 19\end{array}$

B Null geodesic in $\mathrm{AdS}_{5}$-Schwarzschild 20

$\begin{array}{ll}\text { C More on the geometric optics approximation } & 20\end{array}$

D Maximum stopping distance for high-dimension source operators $\quad 21$

D.1 Overview 22

D.2 Quasi-normal modes 25

$\begin{array}{ll}\text { D.3 Saddle point analysis } & 27\end{array}$

D.4 Avoiding WKB exponent divergences 28

\section{Introduction and results}

Various authors [1-4] have made use of gauge-gravity duality to study the stopping distance of massless, high-energy jets in a strongly-coupled plasma of $\mathcal{N}=4$ supersymmetric Yang Mills theory (with and without the addition of fundamental-charge matter). All have found that the furthest that such a jet penetrates through the plasma scales with energy as $E^{1 / 3}$. Most of these methods specified the initial conditions of the problem in the gravity description of the problem, and it is not completely clear exactly what these initial 


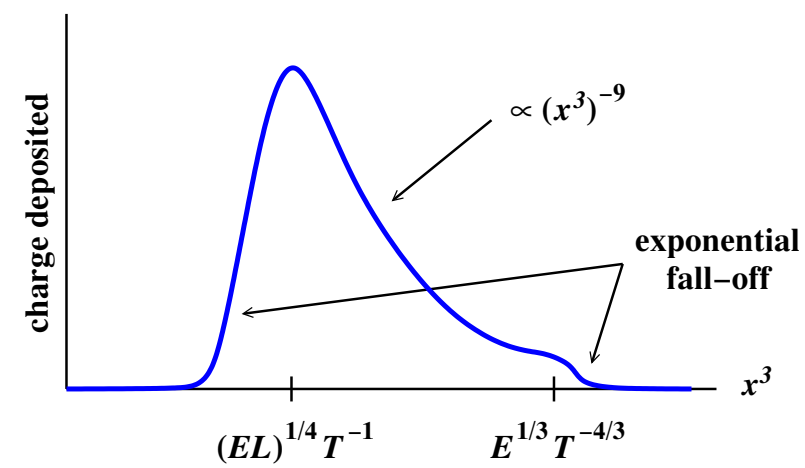

Figure 1. The average deposition of charge as a function of $x^{3}$ for jets created by the source described in section 4.1 and in ref. [4].

conditions correspond to in the gauge theory. However, we recently showed [4] one possible way to set up the entire problem directly in the gauge theory, only then translating to the gravity description using the conventional elements of the AdS/CFT dictionary. We specifically studied jets that carried $\mathrm{R}$ charge, and we measured how far that charge traveled before stopping and thermalizing. Though we did find that the furthest charge would travel through the plasma scaled as $E^{1 / 3}$, we also found that, on average, almost all of our jet's charge stopped and thermalized at a shorter distance that scales as $(E L)^{1 / 4}$, where $L$ is the size of the space-time region where our jet was created. Figure 1 shows a qualitative picture of our result for, on average, how much of our jet's charge was deposited as a function of distance traveled $x^{3}$. (Our convention here is to write 4-dimensional space-time position as $x^{\mu}$ and take our jets to be created near the origin, traveling in the $x^{3}$ direction.) Between the $(E L)^{1 / 4}$ scale and the $E^{1 / 3}$ scale, the distribution falls algebraically like $\left(x^{3}\right)^{-9}$ for jets created by the source used in ref. [4]. We will work in units where $2 \pi T=1$, and in those units the specific formula we derived for figure 1 was

$$
\operatorname{Prob}\left(x^{3}\right) \simeq 2 \frac{\left(4 c^{4} E L\right)^{2}}{\left(2 x^{3}\right)^{9}} \Psi\left(-\frac{c^{4} E L}{\left(2 x^{3}\right)^{4}}\right) \quad \text { for } x^{3} \ll E^{1 / 3}
$$

and

$$
\operatorname{Prob}\left(x^{3}\right) \simeq 4 \frac{\left(c_{2} L\right)^{2}}{E} \Psi(0) \exp \left(-\frac{2 c_{1} x^{3}}{E^{1 / 3}}\right) \quad \text { for } x^{3} \gg E^{1 / 3}
$$

where $\Psi(y)$ is a source-dependent function that suppresses $|y| \gg 1$, causing suppression of $x^{3} \ll(E L)^{1 / 4}$ above. The $c^{\prime}$ s are constants given by

$$
c \equiv \frac{\Gamma^{2}\left(\frac{1}{4}\right)}{(2 \pi)^{1 / 2}},
$$

$c_{1} \simeq 0.927$, and $c_{2} \simeq 3.2$.

The calculation that produced (1.1) was long and not particularly enlightening as to the origin of the $(E L)^{1 / 4}$ scale. The purpose of the current paper is to show how that 
scale, and then the precise result (1.1a) for the case $x^{3} \ll E^{1 / 3}$, can be derived from a very simple calculation of how far a classical massless particle travels in $\mathrm{AdS}_{5}$-Schwarzschild space before falling into the black brane. In the process, we will learn more about exactly what feature of the source determines the $(E L)^{1 / 4}$ scale. We will see that it is not directly the size but the typical "virtuality" $q^{2}$ of the source that matters (where $q^{2} \equiv q_{\mu} \eta^{\mu \nu} q_{\nu}$ is squared 4-momentum).

Our analysis of the distance traveled by falling particles in $\mathrm{AdS}_{5}$-Schwarzschild will be essentially the same as an earlier analysis by Gubser et al. [1] and Chesler et al. [3], who used it in a discussion of the falling endpoint of a classical string. The difference here will be one of context and application: Our analysis of jets [4] does not involve classical strings, and we will use the falling particles to explain the $(E L)^{1 / 4}$ scale.

In our earlier work [4], we created the jet by turning on a small-amplitude source whose space-time dependence had the form

$$
\operatorname{source}(x) \sim e^{i \bar{k} \cdot x} \Lambda_{L}(x)
$$

of (i) a high-energy plane wave $e^{i \bar{k} \cdot x}$ times (ii) a slowly varying envelope function $\Lambda_{L}(x)$ that localizes the source to within a distance $L$ of the origin in both space and time. We took $\bar{k}$ to be light-like:

$$
\bar{k}^{\mu}=(E, 0,0, E) .
$$

In addition, for the sake of simplicity, we took the source to be translation invariant in the two transverse directions. So, for example,

$$
\Lambda_{L}(x)=e^{-\frac{1}{2}\left(x^{0} / L\right)^{2}} e^{-\frac{1}{2}\left(x^{3} / L\right)^{2}} .
$$

The Fourier transform of the source (1.3) is non-negligible in the region of momentum space depicted in figure 2a: a region centered on $\bar{k}$ with width of order $L^{-1}$. We take $L^{-1} \ll E$. Note that this source covers a range of values of $q^{2}$, from 0 to order $\pm E / L$, and the typical size of $\left|q^{2}\right|$ is order $E / L$.

In the gravity description, this source causes a localized perturbation on the boundary of $\mathrm{AdS}_{5}$-Schwarzschild space-time, which then propagates as a wave into the fifth dimension, eventually falling into the black brane horizon. The analysis of jet stopping in refs. $[2,4]$ was based on the analysis of such 5-dimensional waves.

Now imagine instead a source where $\bar{k}$ is slightly off the light-cone,

$$
\bar{k}^{\mu}=(E+\epsilon, 0,0, E-\epsilon)
$$

with $\epsilon \ll E$, and where the envelope size $L$ is wide enough that the picture in momentum space looks like figure $2 \mathrm{~b}$ instead of figure $2 \mathrm{a}$, with the spread $1 / L$ in momenta small compared to $\epsilon$. In this case, the $q^{2}$ of the source is approximately well defined, with $q^{2} \simeq \bar{k}^{2} \simeq-4 E \epsilon$. We will show that in this case the wave created by the boundary perturbation is localized into a small wave packet, whose motion may be approximated by that of a classical, massless particle which starts at the boundary, traveling in the $x^{3}$ direction, with 4-momentum proportional to $q$. The trajectory of such a particle is shown 


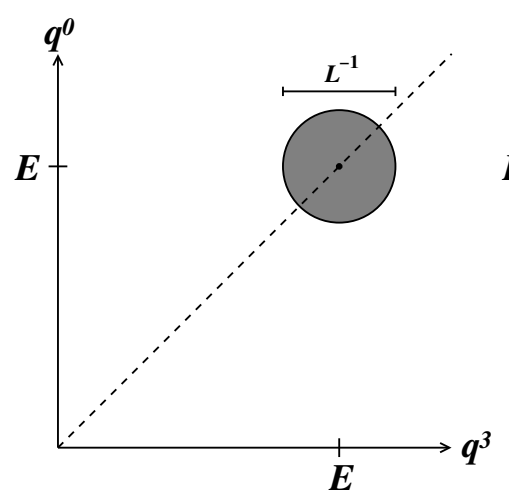

(a)

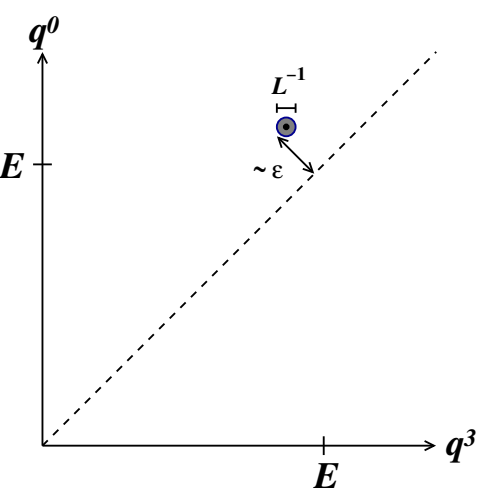

(b)

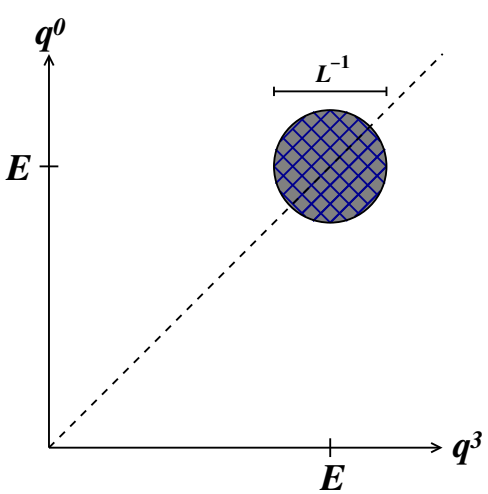

(c)

Figure 2. Qualitative picture of momenta contributing to the source (1.3) used to generate jets (a) for the calculation originally used to find (1.1), with $L^{-1} \ll E$, and (b) in the case $L^{-1} \ll \epsilon \ll E$ of (1.6). Figure (c) depicts (a) as a superposition of distributions of type (b). The cells in (c) that are extremely close to the light cone cannot be treated in particle approximation, but the contribution of cells that can be treated so dominates when $L \ll$ the maximum stopping distance scale $E^{1 / 3}$.

qualitatively in figure 3a. By a simple calculation, we will find that the particle falls into the horizon after covering a distance

$$
x_{\text {stop }}^{3} \simeq \frac{c}{\sqrt{2}}\left(\frac{|\boldsymbol{q}|^{2}}{-q^{2}}\right)^{1 / 4} \simeq \frac{c}{2}\left(\frac{E}{\epsilon}\right)^{1 / 4} .
$$

where the constant $c$ is given by (1.2). As measured by boundary time $x^{0}$, the particle takes an infinite amount of time to fall into the horizon. As it gets closer and closer to the horizon, the boundary distortion that the particle creates (see figure 3b) becomes weaker and more spread out, which corresponds to charge diffusion in the boundary theory after the jet stops and thermalizes. This qualitative picture is similar to the qualitative picture of the effects of a classical string falling into the horizon given in refs. $[1,6]{ }^{1}$

Note that the stopping distance (1.7) only makes sense for $q^{2}<0$ (i.e. $\epsilon>0$ ). The $q^{2}>0$ components of a source do not create an excitation of the system that persists after the source turns off and so are not relevant $[2,4]$.

Now consider the original source of figure $2 \mathrm{a}$ as a superposition of sources like figure $2 \mathrm{~b}$, as depicted in figure 2c. Since sources with different values of $\epsilon$ have different stopping distances (1.7), we might guess that the different pieces of this superposition do not interfere and so the source of figure 2 a simply produces a distribution of stopping distances, weighted by independent probabilities that the source produces a jet with a particular $q^{2}$. That is,

$$
\operatorname{Prob}\left(x^{3}\right) \simeq \int d\left(q^{2}\right) \mathcal{P}\left(q^{2}\right) \delta\left(x^{3}-x_{\text {stop }}^{3}\left(q^{2}\right)\right)
$$

\footnotetext{
${ }^{1}$ See in particular the discussion surrounding figure 2 of ref. [6], which inspired our figure 3b. See also ref. [5].
} 


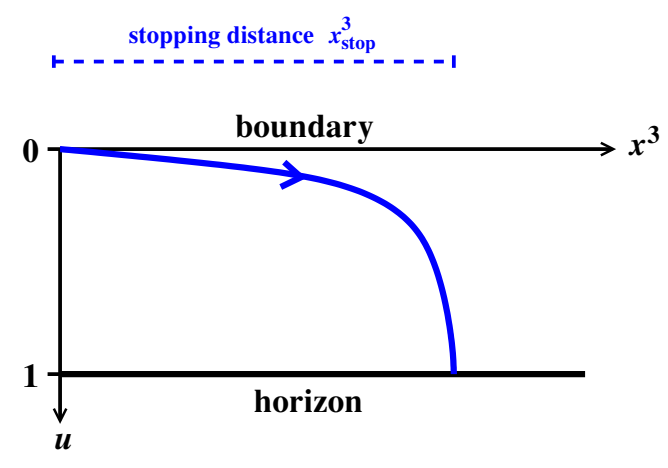

(a)

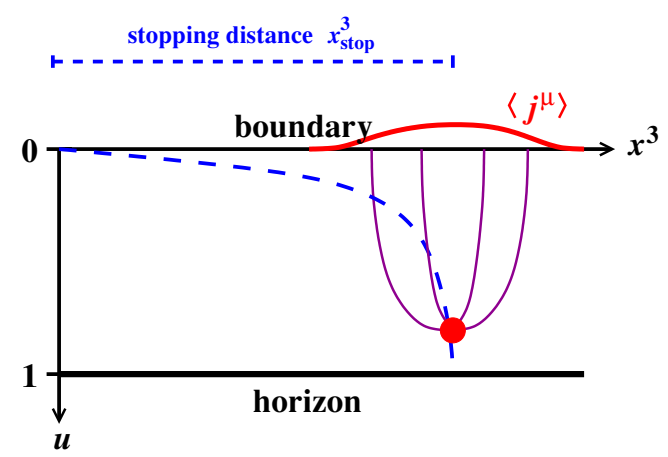

(b)

Figure 3. (a) A classical particle in the $\mathrm{AdS}_{5}$-Schwarzschild space-time, moving in the $x^{3}$ direction as it falls from the boundary to the black brane in the fifth dimension $u$. (b) The presence of the particle (the large dot) perturbs the boundary theory in a manner that spreads out diffusively as the particle approaches the horizon for $x^{0} \rightarrow \infty$.

where $\mathcal{P}\left(q^{2}\right)$ is the probability density for the source to produce a jet with a given $q^{2}$, and where the stopping distance $x_{\text {stop }}^{3}\left(q^{2}\right)$ is given by (1.7). We will verify that this formula precisely reproduces the $x^{3} \ll E^{1 / 3}$ case (1.1a) of our previous result.

We can now see where the $(E L)^{1 / 4}$ scale comes from. It is only time-like source momenta $q^{2}<0$ that produce jets. The typical value of time-like $q^{2}$ for the source of figure $2 \mathrm{a}$ is $q^{2} \sim-E / L$, corresponding to $\epsilon \sim L^{-1}$. Putting this into (1.7), the typical stopping distance in this case is therefore

$$
x_{\text {typical }}^{3} \sim(E L)^{1 / 4} .
$$

Note that it is the $q^{2}$ of the source that determines the stopping distance, and that the typical value of $q^{2}$ is determined by $L$ in the case of figure 2 a.

The estimate (1.9) of the stopping distance ceases to make sense if the size $L$ of the source becomes as large as the stopping distance itself. This happens when

$$
L \sim x_{\text {stop }}^{3} \sim(E L)^{1 / 4}
$$

which gives

$$
x_{\text {stop }}^{3} \sim E^{1 / 3} .
$$

We will see later that this is precisely the case where the wave packet in $\mathrm{AdS}_{5}$-Schwarzschild can no longer be approximated as a particle. The moral is that the simple particle picture gives us not only the $(E L)^{1 / 4}$ scale but also, simply by estimating where it breaks down, the $E^{1 / 3}$ scale as well.

In the next section, we will briefly review the trajectories of massless particles in $\mathrm{AdS}_{5^{-}}$ Schwarzschild and derive the corresponding stopping distance (1.7). In section 3, we discuss the conditions for being able to approximate the 5-dimensional wave problem with particle trajectories and verify that they apply in the case of interest. Then we use the particle picture in section 4 to simply reproduce our original result (1.1a) for charge deposition for 


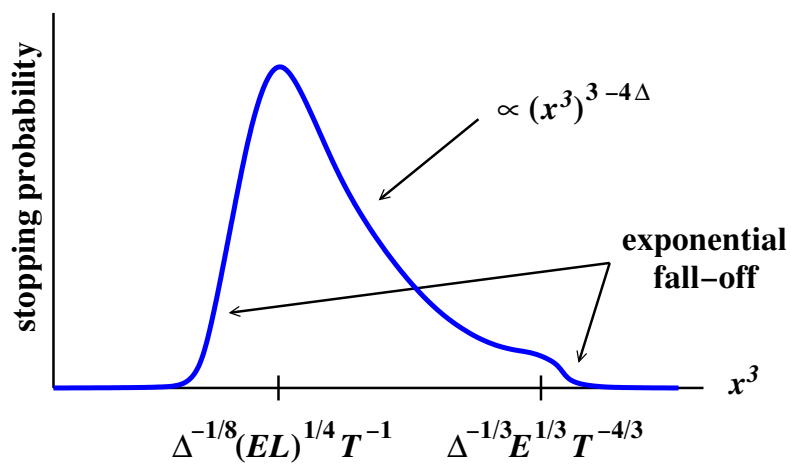

Figure 4. The probability distribution of jet stopping distances for scalar or transverse BPS sources with conformal dimension $\Delta$. Figure 1 corresponds to $\Delta=3$. The scales $x_{\text {typical }}^{3} \sim(E L / \sqrt{\Delta})^{1 / 4}$ and $x_{\max }^{3} \sim(E / \Delta)^{1 / 3}$ indicated along the vertical axis assume that $\Delta$ is held fixed when taking the limit of large energy $E$ (as well as large coupling $g^{2} N_{\mathrm{c}}$ and large $N_{\mathrm{c}}$ ). The parametric scaling with $\Delta$ indicated for $x_{\text {typical }}^{3}$ assumes a Gaussian source envelope (1.5), but the other features shown in the figure are independent of the details of the source envelope.

$x^{3} \ll E^{1 / 3}$. In section 5 , we generalize our results to jets created by other types of source operators than those originally considered in ref. [4]. We will see that figure 1 is modified to figure 4. Finally, we offer our conclusions in section 6 .

As an aside, some readers may be curious how the stopping distance scales $(E L)^{1 / 4}$ and $E^{1 / 3}$ generalize to other dimensions. On the gravity side, it is very easy to generalize the results of this paper to different space-time dimensions $d$ of the boundary, but it is less certain what strongly coupled field theories these classical gravity theories correspond to. (See refs. [7] for proposals.) Ignoring the question of interpretation, we show in appendix A that $(E L)^{1 / 4}$ and $E^{1 / 3}$ generalize to $(E L)^{(d-2) / 2 d}$ and $E^{(d-2) /(d+2)}$ respectively for $d>2$.

In this paper, we will use the convention that Greek indices run over the 4 space-time dimensions $(\mu=0,1,2,3)$ of the boundary theory and capital roman indices run over all five dimensions $(I=0,1,2,3,5)$ of the $\mathrm{AdS}_{5}$-Schwarzschild space-time. The symbol $q^{2}$ will refer to the squared 4-momentum, $q^{2} \equiv q_{\mu} \eta^{\mu \nu} q_{\nu}=-\omega^{2}+|\boldsymbol{q}|^{2}$. When we use light-cone coordinates, our conventions will be

$$
V^{ \pm} \equiv V^{3} \pm V^{0}, \quad V_{ \pm} \equiv \frac{1}{2} V^{\mp}=\frac{1}{2}\left(V^{3} \mp V^{0}\right)
$$

for any 4-vector $V$. Throughout this paper, the adjective "transverse" will refer to the spatial directions 1 and 2 orthogonal to $\boldsymbol{q}$.

\section{Review of falling massless particles}

Null geodesics in a 5-dimensional space with 4-dimensional translation invariance are given by (see appendix B)

$$
x^{\mu}\left(x^{5}\right)=\int \sqrt{g_{55}} d x^{5} \frac{g^{\mu \nu} q_{\nu}}{\left(-q_{\alpha} g^{\alpha \beta} q_{\beta}\right)^{1 / 2}},
$$


where $g$ is the 5 -dimensional metric and $q_{I}$ is a constant of motion for $I=0,1,2,3$. We will work in coordinates where the metric is ${ }^{2}$

$$
d s^{2}=\frac{R^{2}}{4}\left[\frac{1}{u}\left(-f d t^{2}+d \boldsymbol{x}^{2}\right)+\frac{1}{u^{2} f} d u^{2}\right],
$$

where $f \equiv 1-u^{2}$, and $R$ is the $\mathrm{AdS}_{5}$ radius. The boundary is at $u=0$ and the horizon at $u=1$. If we take the 3 -momentum $\boldsymbol{q}$ to point in the $x^{3}$ direction, writing $q_{\mu}=(-\omega, 0,0,|\boldsymbol{q}|)$, then (2.1) gives the total distance $x^{3}$ traveled in falling from the boundary to the horizon to be

$$
x_{\text {stop }}^{3}=\int_{0}^{1} \frac{d u}{\sqrt{u\left(u^{2}-\frac{q^{2}}{|\boldsymbol{q}|^{2}}\right)}},
$$

where $q^{2} \equiv q_{\mu} \eta^{\mu \nu} q_{\nu}$ is the flat-space square of the 4-momentum. This is the same result as refs. $[1,3] .^{3}$

Now let us apply this result to the case $\left|q^{2}\right| \ll|\boldsymbol{q}|^{2} \simeq E^{2}$ relevant to the source of figure $2 \mathrm{~b}$. For small $-q^{2}$, the integral of $(2.3)$ is dominated by small $u$, and so we may approximate

$$
x_{\text {stop }}^{3} \simeq \int_{0}^{\infty} \frac{d u}{\sqrt{u\left(u^{2}-\frac{q^{2}}{|\boldsymbol{q}|^{2}}\right)}}=\frac{c}{\sqrt{2}}\left(\frac{|\boldsymbol{q}|^{2}}{-q^{2}}\right)^{1 / 4}
$$

which gives (1.7).

\section{$3 \quad$ Wave packets and geometric optics}

In this section, we will discuss the conditions necessary for making the particle approximation. A wave packet behaves like a particle when it is wide enough to contain many phase oscillations of the field yet small enough that the properties of the background do not vary significantly across its width, as depicted in figure $5 \mathrm{~b}$ at a particular moment in time. We can arrange such a width provided the background properties do not vary significantly over one wavelength of the phase oscillation. This is the geometric optics limit, which we referred to in our earlier work [4] as a WKB approximation. To check the geometric optics limit, one may focus as in figure $5 \mathrm{c}$ on a wave with a single, generic value of $q$ typical of the wave packet, and investigate how much things change over one phase oscillation.

To assess whether a wave packet is adequately particle-like to use a particle-based calculation of the stopping distance, it will be helpful to understand what the important scale for $u$ is in determining the stopping distance (2.4). The integral in (2.4) is dominated by $u$ of order

$$
u_{\star} \sim \sqrt{\frac{-q^{2}}{|\boldsymbol{q}|^{2}}} \sim \sqrt{\frac{\epsilon}{E}} .
$$

\footnotetext{
${ }^{2}$ Our formulas in this paper would be a little tidier (fewer square roots) if we worked with the coordinate $z \equiv 2 \sqrt{u}$ instead of $u$. We will stick with $u$ in order to facilitate comparison with our previous work [4].

${ }^{3}$ Our (2.4) corresponds to the first part of eq. (5.3) of Gubser et al. [1], where their $y$ is our $\sqrt{u}$, their $z_{H}$ is 2 in our units $2 \pi T=1$, their $p_{1} / p_{0}$ is replaced by the $q_{3} / q_{0}$ of the momentum $q_{\mu}$ typical of our source, and their $y_{\mathrm{UV}}$ is set to zero. It also corresponds to eq. (4.28) of Chesler et al. [3], where their $u$ is our $2 \sqrt{u}$, their $u_{h}=2$ in our units, their $\xi$ is replaced by our $q_{0} / q_{1}$, and their $u_{*}$ is set to zero.
} 


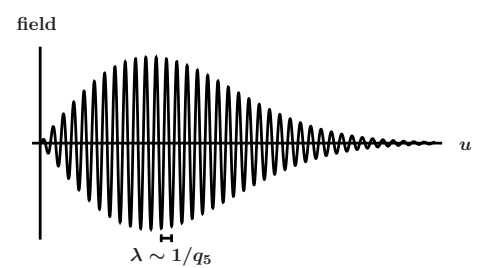

(a)

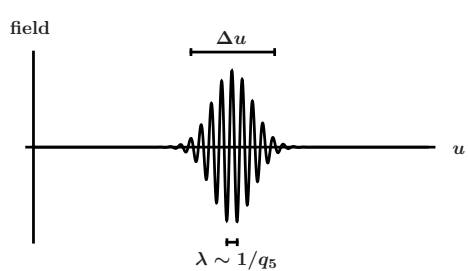

(b)

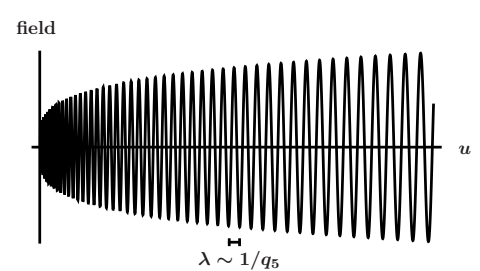

(c)

Figure 5. (a,b) A snapshot in time $x^{0}$ of waves in the fifth dimension $u$ for times after the boundary source has turned off but early enough that $u \lesssim u_{\star} \ll 1$ (that is, before the wave gets very close to the horizon). (a) shows the type of wave generated by a localized source that superposes a range of $q^{2}$ values such as figure $2 \mathrm{a}$. (b) shows the wave packet generated by a source with approximately well-defined $q^{2}$ such as figure $2 \mathrm{~b}$. (c) shows a single 4-momentum component, corresponding to a single, definite value of 4-momentum $q_{\mu}$. Case (a) matches figure 7a of our earlier paper [4].

The relevant question is then whether the background varies significantly across one phase oscillation for $u \sim u_{\star}$. By (1.7), the distances $x_{\text {stop }}^{3} \lesssim E^{1 / 3}$ relevant to jet stopping correspond to $\epsilon \gtrsim E^{-1 / 3}$ in the particle picture and so to $u_{\star} \lesssim E^{-2 / 3} \ll 1$. So we may focus on small $u$ in what follows.

\subsection{Geometric optics}

For a massless 5-dimensional field with definite 4-momentum $q_{\mu}$, the exponential in the WKB approximation is

$$
\exp \left(i q_{\mu} x^{\mu}+i \int d x^{5} q_{5}\left(x^{5}\right)\right)
$$

where the $q_{\mu}$ are constant and $q_{5}\left(x^{5}\right)$ is determined by the 5-dimensional massless condition $q_{I} g^{I J} q_{J}=0$, giving $^{4}$

$$
q_{5}=\sqrt{g_{55}\left(-q_{\mu} g^{\mu \nu} q_{\nu}\right)}
$$

For the metric $(2.2)$, this is

$$
q_{5}(u)=\frac{1}{f} \sqrt{\frac{u^{2}|\boldsymbol{q}|^{2}-q^{2}}{u}} .
$$

For the geometric optics limit, we need the wavelength to vary insignificantly over one wavelength. ${ }^{5}$ For the important values $u \sim u_{\star} \ll 1$ of $u$, the wavelength $\lambda(u) \sim 1 / q_{5}(u)$ in the fifth dimension satisfies this condition if

$$
u_{\star} q_{5}\left(u_{\star}\right) \gg 1
$$

\footnotetext{
${ }^{4}$ The null geodesics $(2.1)$ can be expressed in terms of $q_{5}$ as $x^{\mu}\left(x^{5}\right)=-\int d x^{5}\left(\partial q_{5} / \partial q_{\mu}\right)$. This particle formula is simply the saddle point condition $\partial_{q_{\mu}}\left[i q_{\nu} x^{\nu}+i \int d x^{5} q_{5}\left(x^{5}\right)\right]=0$ with respect to $q_{\mu}$ for the wave (3.2). Also, $\int d x^{5} q_{5}\left(x^{5}\right)$ was referred to as the WKB exponent $S$ in ref. [4], where various expansions of the integral may be found.

${ }^{5}$ This condition can be phrased in an $x^{5}$-reparametrization invariant way as $\nabla_{5}\left(1 / q_{5}\right) \ll 1$, which is $\left(g_{55}\right)^{-1 / 2} \partial_{5}\left[\left(g_{55}\right)^{1 / 2} / q_{5}\right] \ll 1$. We give more detail in appendix $\mathrm{C}$, in the specific context of the particular type of source operator that we used in our original calculation.
} 
which, using (3.4), is ${ }^{6}$

$$
u_{\star} \gg \frac{1}{-q^{2}} .
$$

Using the size (3.1) of $u_{\star}$, this condition is $\left(-q^{2}\right)^{3} /|\boldsymbol{q}|^{2} \gg 1$, or equivalently

$$
\epsilon \gg E^{-1 / 3} \text {. }
$$

Referring to the stopping distance (1.7), we then see that the approximation of geometric optics, necessary for a particle interpretation, breaks down unless

$$
x_{\text {stop }}^{3} \ll E^{1 / 3} .
$$

Within the context of our approach to jet stopping, a proper analysis of what happens at distances $\gtrsim E^{1 / 3}$ requires a wave rather than particle description of the problem, as in refs. $[2,4]$. The wave analysis gives exponential fall-off for propagation beyond $E^{1 / 3}$, as described by (1.1b).

More on the geometric optics approximation can be found in appendix C.

\subsection{Wave packets}

The geometric optics limit for (3.8) allows us to create localized wave packets. Here we will see how wide those wave packets are in $u$ for sources of the form of figure $2 \mathrm{~b}$. We are primarily interested in the case where the center of the wave packet is at the critical scale $u \sim u_{\star}$ in the fifth dimension. However, the presentation will be a little more straightforward if we first make parametric estimates for earlier times, when the center of the wave packet is at $u \ll u_{\star}$, and then extrapolate those parametric estimates to $u \sim u_{\star}$.

For $u \ll u_{\star}$, the distance traveled (2.1) for a massless particle is

$$
x^{3}(u)=\int_{0}^{u} \frac{d u^{\prime}}{\sqrt{u^{\prime}\left(u^{\prime 2}-\frac{q^{2}}{|\boldsymbol{q}|^{2}}\right)}} \simeq 2 \sqrt{\frac{u|\boldsymbol{q}|^{2}}{-q^{2}}} \simeq \sqrt{\frac{u E}{\epsilon}} .
$$

Turning this around, the location of the particle in the fifth dimension is

$$
u \simeq \frac{\epsilon\left(x^{3}\right)^{2}}{E} .
$$

When the particle is replaced by a wave packet, there are two sources of uncertainty. The size $L$ of the source introduces an uncertainty in the initial position of the excitation of $\Delta x^{3} \sim L$. It also introduces an uncertainty in the 4-momentum $q$, and so in $\epsilon$, of $\Delta \epsilon \sim 1 / L$ as in figure $2 \mathrm{~b}$. From (3.10), the combined uncertainty $\Delta u$ in $u$ is then of order

$$
\frac{\Delta u}{u} \sim \max \left(\frac{\Delta \epsilon}{\epsilon}, \frac{\Delta x^{3}}{x^{3}}\right) \sim \max \left(\frac{1}{L \epsilon}, L \sqrt{\frac{\epsilon}{u E}}\right) .
$$

Extrapolating this parametric estimate to the case $u \sim u_{\star}$ of interest, (3.1) and (3.11) give

$$
\left(\frac{\Delta u}{u}\right)_{\star} \sim \max \left(\frac{1}{L \epsilon}, L\left(\frac{\epsilon}{E}\right)^{1 / 4}\right) .
$$

\footnotetext{
${ }^{6}$ In the language of ref. [4], the condition $u_{\star} \gg 1 /\left(-q^{2}\right)$ is $u_{\star} \gg u_{\text {match }}$.
} 
The wave packet will then be localized provided (i) $L \gg 1 / \epsilon$, as in figure $2 \mathrm{~b}$, and (ii) $L \ll(E / \epsilon)^{1 / 4}$. By (1.7), the last condition is just the condition that $L$ be much less than the stopping distance $x_{\text {stop }}^{3}$.

\section{Reproducing the distribution of stopping distances}

Now we will show that the formula (1.1a) found in our earlier work [4] for the average distribution of charge deposition can be understood as a convolution (1.8) of the particle stopping distance with the probability density $\mathcal{P}\left(q^{2}\right)$ for the source to create a jet with a given $q^{2}$.

We will later give in section 5 a very general argument, based on dimensional analysis, for determining the $\mathcal{P}\left(q^{2}\right)$ associated with different choices of source operator. This argument will also require a discussion of massive fields and massive particles in the gravity dual. For the moment, we will be less general, stick to the specific type of source operator that we used in previous work, and show how to extract $\mathcal{P}\left(q^{2}\right)$ from a result for the average total charge produced by the operator. Readers who would prefer to just see the more general argument may skip section 4.1 below and instead wait for section 5.2.

\subsection{Extracting $\mathcal{P}\left(q^{2}\right)$ from results in the literature}

In ref. [4], we used a source involving R-current operators $j_{\mu}^{a}$. Specifically, we modified the 4-dimensional gauge theory Lagrangian by

$$
\mathcal{L} \rightarrow \mathcal{L}+j_{\mu}^{a} A_{\mathrm{cl}}^{a \mu}
$$

with a localized background field

$$
A_{\mathrm{cl}}^{\mu}(x)=\bar{\varepsilon}^{\mu} \mathcal{N}_{A}\left[\frac{\tau^{+}}{2} e^{i \bar{k} \cdot x}+\text { h.c. }\right] \Lambda_{L}(x),
$$

where $\mathcal{N}_{A}$ is an arbitrarily small source amplitude, $\bar{\varepsilon}$ is a transverse linear polarization, and $\tau^{i}$ are Pauli matrices for any $\mathrm{SU}(2)$ subgroup of the $\mathrm{SU}(4) \mathrm{R}$-symmetry. We then measured the response $\left\langle j^{(3) \mu}(x)\right\rangle$ of the $\mathrm{R}$ charge current associated with $\tau^{3} / 2$. The gravity dual to the $\mathrm{R}$ charge current operators is a massless 5-dimensional $\mathrm{SU}(4)$ gauge field. We chose our source (4.2) to be translationally invariant in the transverse spatial directions $\left(x^{1}, x^{2}\right)$ to simplify the calculation. In what follows, we will refer to the $\mathrm{R}$ charge associated with $\tau^{3} / 2$ as simply "the charge."

For an arbitrarily small source amplitude $\mathcal{N}_{A}$, the source will usually have no effect at all on the system. On rare occasions, with probability proportional to $\mathcal{N}_{A}^{2}$, the source creates an excitation (in our case a "jet") with the same quantum numbers as the source operator. In our case (4.2), that means it creates a jet with total charge equal to 1 . The creation of an excitation with different quantum numbers would be even higher-order in $\mathcal{N}_{A}$ and so negligible. Since an excitation (if any is created) has charge 1, the average total charge $\mathcal{Q}$ created by the source is then equal to the probability of the source creating a jet. We will see that from the previously calculated result for $\mathcal{Q}$ we can then extract the probability density $\mathcal{P}\left(q^{2}\right)$. 
In ref. $[4],{ }^{7}$ we showed how to use the field theory Ward identity to make a simple calculation of the average charge $\mathcal{Q}$. Here we will just quote the result, which was

$$
\mathcal{Q} \simeq \frac{2 \pi \mathcal{N}_{A}^{2}}{g_{\mathrm{SG}}^{2}} \int \frac{d^{4} q}{(2 \pi)^{4}} \theta\left(-q^{2}\right)\left|q^{2} \| \tilde{\Lambda}_{L}(q-\bar{k})\right|^{2},
$$

where $g_{\mathrm{SG}}^{2}=4 \pi / N_{\mathrm{c}}\left(N_{\mathrm{c}} \rightarrow \infty\right.$ is the number of colors $)$ and $\theta$ is the step function. The $\tilde{\Lambda}_{L}(q-\bar{k})$ in $(4.3)$ is simply the Fourier transform of the $x$-dependence (1.3) of the source. Our source only has support for $q=\bar{k}+\Delta q$ with $\Delta q$ small compared to $\bar{k}$, in which case $q^{2} \simeq 4 E q_{+}$. Eq. (4.3) may then be approximated as

$$
\mathcal{Q} \simeq \frac{8 \pi E \mathcal{N}_{A}^{2}}{g_{\mathrm{SG}}^{2}} V_{\perp} \int \frac{2 d q_{+} d \Delta q_{-}}{(2 \pi)^{2}} \theta\left(-q_{+}\right)\left|q_{+}\right|\left|\tilde{\Lambda}_{L}^{(2)}\left(q_{+}, \Delta q_{-}\right)\right|^{2}
$$

where $\tilde{\Lambda}_{L}^{(2)}$ is the two-dimensional Fourier transform

$$
\tilde{\Lambda}_{L}^{(2)}\left(q_{+}, q_{-}\right)=\int \frac{d x^{+} d x^{-}}{2} \Lambda_{L}(x) e^{-i\left(q_{+} x^{+}+q_{-} x^{-}\right)}
$$

of the source envelope and $V_{\perp}$ is the area of transverse space $\left(x^{1}\right.$ and $\left.x^{2}\right)$. Since (4.4) involves an integral over $q_{+}$, and since $\mathcal{Q}$ is the total probability of creating a jet, it is natural to interpret (4.4) as giving a probability density

$$
P_{+}\left(q_{+}\right) \simeq \frac{8 \pi E \mathcal{N}_{A}^{2}}{g_{\mathrm{SG}}^{2}} V_{\perp} \int \frac{2 d \Delta q_{-}}{(2 \pi)^{2}} \theta\left(-q_{+}\right)\left|q_{+}\right|\left|\tilde{\Lambda}_{L}^{(2)}\left(q_{+}, \Delta q_{-}\right)\right|^{2}
$$

for producing a jet with a given value of $q_{+}$, with $\mathcal{Q}=\int d q_{+} P_{+}\left(q_{+}\right)$. If a jet $i s$ produced, the probability distribution for its $q_{+}$is then the relative probability

$$
\mathcal{P}_{+}\left(\bar{q}_{+}\right) \equiv \frac{P_{+}\left(\bar{q}_{+}\right)}{\mathcal{Q}} \simeq \frac{\theta\left(-\bar{q}_{+}\right)\left|\bar{q}_{+}\right| \int d \Delta q_{-}\left|\tilde{\Lambda}_{L}^{(2)}\left(\bar{q}_{+}, \Delta q_{-}\right)\right|^{2}}{\int d q_{+} d \Delta q_{-} \theta\left(-q_{+}\right)\left|q_{+}\right|\left|\tilde{\Lambda}_{L}^{(2)}\left(q_{+}, \Delta q_{-}\right)\right|^{2}} .
$$

Here we've put a bar over the argument of $P_{+}$just to distinguish it from the $q_{+}$integration variable in the denominator on the right-hand side. Now package the source dependence into the definition

$$
\Psi\left(\bar{q}_{+} L\right) \equiv \frac{\int d q_{-}\left|\tilde{\Lambda}_{L}^{(2)}\left(\bar{q}_{+}, q_{-}\right)\right|^{2}}{4 L^{2} \int d q_{+} d q_{-} \theta\left(-q_{+}\right)\left|q_{+}\right|\left|\tilde{\Lambda}_{L}^{(2)}\left(q_{+}, q_{-}\right)\right|^{2}} .
$$

This is the definition we made in our earlier work [4] for the $\Psi$ that appears in the charge deposition result (1.1). With this definition,

$$
\mathcal{P}_{+}\left(q_{+}\right)=4 L^{2} \theta\left(-q_{+}\right)\left|q_{+}\right| \Psi\left(q_{+} L\right) .
$$

\footnotetext{
${ }^{7}$ See specifically appendix A of ref. [4].
} 


\subsection{Using $\mathcal{P}\left(q^{2}\right)$ to get $\operatorname{Prob}\left(x^{3}\right)$}

Since $q^{2} \simeq 4 E q_{+}$, the probability distribution $\mathcal{P}\left(q^{2}\right)$ for $q^{2}$ is related to the probability distribution $\mathcal{P}_{+}\left(q_{+}\right)$of (4.9) for $q_{+}$by

$$
\mathcal{P}\left(q^{2}\right) \simeq \frac{1}{4 E} \mathcal{P}_{+}\left(\frac{q^{2}}{4 E}\right) .
$$

However, at this point it will be easier to just stick with $q_{+}$and $\mathcal{P}_{+}$.

Note that in each cell of figure $2 \mathrm{c}$, the typical value of $q_{+}$is just what we have previously called $-\epsilon$ for that cell. The distribution (1.8) of stopping distances based upon the picture of massless falling particles in 5 dimensions can then be written as

$$
\begin{aligned}
\operatorname{Prob}\left(x^{3}\right) & \simeq \int d \epsilon \mathcal{P}_{+}(-\epsilon) \delta\left(x^{3}-x_{\text {stop }}^{3}(\epsilon)\right) \\
& \simeq 4 L^{2} \int_{0}^{\infty} d \epsilon \epsilon \Psi(-L \epsilon) \delta\left(x^{3}-\frac{c}{2}\left(\frac{E}{\epsilon}\right)^{1 / 4}\right) \\
& =2 \frac{\left(4 c^{4} E L\right)^{2}}{\left(2 x^{3}\right)^{9}} \Psi\left(-\frac{c^{4} E L}{\left(2 x^{3}\right)^{4}}\right) .
\end{aligned}
$$

As promised, in the case $x^{3} \ll E^{1 / 3}$ where we have argued that the particle picture should work, this exactly reproduces our earlier result (1.1a) that came from a full, much more complicated calculation.

\section{Massive particles in 5 dimensions}

In the preceding sections, we have assumed that the 5-dimensional bulk field dual to the source which creates the jet is massless, such as the 5-dimensional gauge field dual to $\mathrm{R}$ current operators. One may wonder what results change if we choose different types of source operators that are instead dual to massive bulk fields. In this section, we will see that the basic qualitative picture of figure 1 of the distribution of stopping distances remains the same, except that the exponent of the $\left(x^{3}\right)^{-9}$ power-law tail changes, depending on the conformal dimension of the source operator.

When we wish to make contact with a particular example, we will for simplicity restrict attention to scalar BPS (e.g. chiral primary [8]) operators. ${ }^{8}$ In that case, the mass $m$ of the 5 -dimensional field is related to the scaling dimension $\Delta$ of the operator by [9]

$$
(R m)^{2}=\Delta(\Delta-d)
$$

where $R$ is the radius of $\mathrm{AdS}_{5}$, and $d=4$ is the dimension of ordinary space-time. The possible values of $\Delta$ are bounded below by

$$
\Delta \geq \frac{d}{2}
$$

\footnotetext{
${ }^{8}$ Examples of scalar BPS operators include the Lagrangian density and the symmetrized trace $\operatorname{tr}\left(\phi_{\left(i_{1}\right.} \phi_{i_{2}} \cdots \phi_{i_{n}}\right)$, where $\phi_{1}, \phi_{2}$, and $\phi_{3}$ are the three complex scalar fields of $\mathcal{N}=4$ supersymmetric Yang Mills.
} 
In what follows, we will hold $m$ fixed when we consider the limit of large jet energy $E$. However, it will be interesting to consider the case $\Delta \gg 1$ (i.e. $R m \gg 1$ ) in addition to the case where $\Delta$ is of order one. We will find that the typical and maximum stopping distances decrease for larger $\Delta$.

We will study the propagation of excitations of a massive bulk field by studying the propagation of massive particles in the bulk, similar to the massless case studied earlier in this paper. (An alternative discussion directly in terms of a wave analysis is sketched in appendix D.) We should emphasize that the term "massive" refers only to the bulk fields and corresponding bulk "particles" in our discussion, and so to the conformal dimension of the source operators in the boundary theory. We have not introduced any masses in the 4-dimensional strongly-coupled field theory: the theory is still just $\mathcal{N}=4$ supersymmetric Yang Mills theory.

\subsection{Stopping distance of massive particles}

For a particle of mass $m$ in 5 dimensions, the stopping distance integrals (2.1) and (2.3) are modified to

$$
x_{\text {stop }}^{3}=\int \sqrt{g_{55}} d x^{5} \frac{g^{\mu \nu} q_{\nu}}{\left(-q_{\alpha} g^{\alpha \beta} q_{\beta}-m^{2}\right)^{1 / 2}}=\int \frac{d u}{\sqrt{u\left(u^{2}-\frac{q^{2}}{|\boldsymbol{q}|^{2}}\right)-\frac{(R m)^{2}}{4|\boldsymbol{q}|^{2}} f}} .
$$

As in the massless case, we shall see below that the stopping distance will be dominated by $u \sim u_{\star} \ll 1$. So we will be able to approximate $f \simeq 1$ above:

$$
x_{\text {stop }}^{3} \simeq \int \frac{d u}{\sqrt{u\left(u^{2}-\frac{q^{2}}{|\boldsymbol{q}|^{2}}\right)-\frac{(R m)^{2}}{4|\boldsymbol{q}|^{2}}}} .
$$

For $\Delta>d$ (in which case $m^{2}$ is positive), there is an issue with the lower limit of integration in (5.4): our classical particle with 4-momentum $q_{\mu}$ cannot exist in the region where the square root in (5.4) is imaginary. For $u \ll u_{*}$ (and focusing on $q^{2}<0$ ), this condition allows for a classical particle when $u \geq u_{\min }$ with

$$
u_{\text {min }} \simeq \frac{(R m)^{2}}{-4 q^{2}}
$$

How to interpret this? The wave equation is not well described by geometric optics near the turning point $u_{\min }$. However, as long as $u_{\min } \ll u_{\star}$, the calculation of the stopping distance will be dominated by much larger $u$ (where $m$ is ignorable), and so we may still use the particle picture to approximate

$$
x_{\text {stop }}^{3} \simeq \int_{\sim u_{\min }}^{1} \frac{d u}{\sqrt{u\left(u^{2}-\frac{q^{2}}{|\boldsymbol{q}|^{2}}\right)-\frac{1}{4}(R m)^{2}}} \simeq \int_{0}^{\infty} \frac{d u}{\sqrt{u\left(u^{2}-\frac{q^{2}}{|\boldsymbol{q}|^{2}}\right)}},
$$

which is the same as the massless particle result (2.4). In this respect, the mass can be ignored. 
What happens at $u \sim u_{\min }$ can be made more concrete by returning to the wave problem and looking at the solution to the massive scalar wave equation

$$
\frac{1}{\sqrt{-g}} \partial_{5}\left(\sqrt{-g} g^{55} \partial_{5} \Phi\right)=\left(q_{\mu} g^{\mu \nu} q_{\nu}+m^{2}\right) \Phi
$$

in the limit $u \ll u_{\star} \ll 1$. In this limit, one is close enough to the boundary that $\mathrm{AdS}_{5^{-}}$ Schwarzschild is approximately just $\mathrm{AdS}_{5}$, and the equation becomes the zero-temperature wave equation of a massive scalar in $\mathrm{AdS}_{5}$. The retarded solution to this equation is

$$
\Phi \simeq \mathcal{N}_{q} \frac{i \pi}{\Gamma(\nu)}\left(\frac{1}{2} \sqrt{-q^{2}}\right)^{\nu}(4 u)^{d / 4} H_{\nu}^{(1)}\left(\sqrt{-4 u q^{2}}\right)
$$

where $H_{\nu}^{(1)}$ is the Hankel function, $d=4$ is the space-time dimension of the boundary theory, $\mathcal{N}_{q}$ is an overall normalization, and

$$
\nu=\Delta-\frac{d}{2}
$$

The solution behaves like

$$
\Phi \simeq \mathcal{N}_{q} z^{d-\Delta}
$$

in the boundary limit $z \rightarrow 0$, where $z \equiv 2 \sqrt{u}$. The divergence of (5.10) as $z \rightarrow 0$ for $\Delta>d$ (i.e. $m^{2}>0$ ) reflects the renormalization required of the corresponding operators in the 4-dimensional gauge theory. In our discussion, we will be able to ignore the details of holographic renormalization prescriptions and simply summarize that (5.8) should approach $z^{d-\Delta} \phi_{\mathrm{b}}(q)$ as $z$ approaches the (regulated) boundary, where $\phi_{\mathrm{b}}$ is the (renormalized) boundary source. Choosing $\phi_{\mathrm{b}}(q)=1$ defines the bulk-to-boundary propagator, which corresponds to (5.8) with $\mathcal{N}_{q} \simeq 1 .^{9}$

The Hankel function goes through many oscillations, and so is well approximated by the geometric optics limit, when its argument is large compared to both 1 and $\nu$. In our case, this condition is parametrically equivalent to

$$
u \gg u_{\text {match }} \equiv \frac{\max \left(1,(R m)^{2}\right)}{-q^{2}},
$$

which may be also be written as

$$
u \gg \max \left(\frac{1}{-q^{2}}, u_{\min }\right) .
$$

This generalizes the condition $u \gg 1 /\left(-q^{2}\right)$ previously discussed for the massless case. If we convolve (5.8) with a high-energy source (1.3), we will not be able to use the particle

\footnotetext{
${ }^{9}$ In more detail, follow ref. [10] and normalize the bulk-to-boundary propagator to be $z_{\mathrm{B}}^{d-\Delta}$ at $z=z_{\mathrm{B}}$, where $z_{\mathrm{B}}$ is arbitrarily small. Then

$$
\mathcal{N}_{q}=z_{\mathrm{B}}^{d-\Delta}\left[\frac{i \pi}{\Gamma(\nu)}\left(\frac{1}{2} \sqrt{-q^{2}}\right)^{\nu}\left(4 u_{\mathrm{B}}\right)^{d / 4} H_{\nu}^{(1)}\left(\sqrt{-4 u_{\mathrm{B}} q^{2}}\right)\right]^{-1}=1+O\left(z_{\mathrm{B}}\right)
$$

and one takes $z_{\mathrm{B}} \rightarrow 0$ at the very end of the calculation. In yet more detail, a systematic method for holographic renormalization is described in refs. [11-13].
} 
approximation to figure out the details of what is happening at $u \sim u_{\min }$, but we will be able to use it when the resulting wave packet propagates to $u \sim u_{\star}$ provided $u_{*} \gg u_{\text {match }}$, and so we may then use the particle approximation to calculate the stopping distance.

Because we get the same stopping distance as for the massless case, we can take over (1.7):

$$
x_{\text {stop }}^{3} \simeq \frac{c}{\sqrt{2}}\left(\frac{|\boldsymbol{q}|^{2}}{-q^{2}}\right)^{1 / 4} \simeq \frac{c}{2}\left(\frac{E}{\epsilon}\right)^{1 / 4} .
$$

The geometric optics approximation at $u \sim u_{\star}$ (and so this result for the stopping distance) will fail unless $u_{\text {match }} \ll u_{\star}$. Using (3.1) and (5.11), that condition requires

$$
\epsilon \gg\left(\frac{E}{\max \left(1,(R m)^{4}\right)}\right)^{-1 / 3} \text {. }
$$

One might suspect that the particle approximation breaks down at the maximum possible stopping distance, in which case (5.13) then gives that maximum to be

$$
x_{\max }^{3} \sim\left(\frac{E}{\max (1, R m)}\right)^{1 / 3} \sim\left(\frac{E}{\Delta}\right)^{1 / 3} .
$$

We give a more detailed argument for this result in appendix D.

Eq. (5.15) implies that the maximum stopping distance decreases as the conformal dimension $\Delta$ of the BPS source operator is increased. This qualitative feature is not novel to the strongly-coupled theory: it is true for the weakly-coupled theory as well. For the BPS operators, large $\Delta$ corresponds to an operator with roughly $\Delta$ powers of scalar fields, such as $\operatorname{tr}\left(\phi^{\Delta}\right)$, where $\phi$ is one of the three complex scalar fields in the theory. In weak coupling, if we inject total energy $E$ with such an operator, it will typically generate $\Delta$ particles that each have energy of order $E / \Delta$. In weak coupling, the stopping distance of a particle with energy $E$ scales as $E^{1 / 2}$ (up to logarithms), and so the stopping distance of the $\Delta$ particles each with energy $E / \Delta$ will scale as $x_{\max }^{3} \sim(E / \Delta)^{1 / 2}$.

\subsection{Generalizing the power-law tail}

In this section, we investigate how the $\left(x^{3}\right)^{-9}$ power-law tail in figure 1 generalizes to other choices of source operator. We will take the source term in the gauge-theory action to be of the form

$$
\int_{x} e^{i \bar{k} \cdot x} \Lambda_{L}(x) \mathcal{O}(x)
$$

where $\mathcal{O}(x)$ is a scalar BPS operator with dimension $\Delta$. We will see, however, that our result also applies to the case of $\mathcal{O}$ being a transverse-polarized $\mathrm{R}$ current, which was the case discussed in section 4 .

Since the dependence (5.13) of the stopping distance on $q^{2}$ is the same as in the massless case, the only significant qualitative difference in the distribution of stopping distances will come from the distribution $\mathcal{P}\left(q^{2}\right)$ of $q^{2}$ created by the source operator. The shape of this distribution is determined by the dimension $\Delta$ of the source operator, as we now describe. As discussed in refs. [2, 4], temperature does not affect the initial creation of the jet, and 
so we can simplify the analysis by evaluating $\mathcal{P}\left(q^{2}\right)$ at zero temperature. Consider the probability density in $q$ associated with a source operator $\mathcal{O}$ acting on the vacuum:

$$
\begin{aligned}
\sum_{\text {any }}\left\langle\operatorname{any}\left|[\mathcal{O}(q)]^{\dagger}\right| \operatorname{vac}\right\rangle^{*}\left\langle\operatorname{any}\left|\left[\mathcal{O}\left(q^{\prime}\right)\right]^{\dagger}\right| \text { vac }\right\rangle & \\
& =\left\langle\operatorname{vac}\left|\mathcal{O}(q)\left[\mathcal{O}\left(q^{\prime}\right)\right]^{\dagger}\right| \operatorname{vac}\right\rangle \equiv i G_{>}(q)(2 \pi)^{d} \delta^{(d)}\left(q-q^{\prime}\right),
\end{aligned}
$$

where $G_{>}$is the Wightman correlator of $\mathcal{O}$. At zero temperature, it is related to the spectral density $\rho$ of the operator by ${ }^{10}$

$$
i G_{>}(q)=\theta\left(q^{0}\right) \rho(q) .
$$

The distribution of jet 4-momenta is therefore given by the spectral density $\rho(q)$. At zero temperature, Lorentz and scaling invariance allow us to use simple dimensional analysis to know how $\rho$ scales with $q:^{11}$

$$
\rho(q) \propto \theta\left(-q^{2}\right)\left(-q^{2}\right)^{\nu},
$$

where $\nu=\Delta-\frac{1}{2} d$ as in (5.9). The $\theta\left(-q^{2}\right)$ appears because only sources with time-like $q^{2}$ produce persistent excitations at zero temperature.

If $\mathcal{O}$ were a vector operator $V^{\mu}$, like an $\mathrm{R}$ current, the dimensional analysis would be complicated by the fact that one could get factors of $q^{\mu}$ associated with the vector index (rather than only factors of the virtuality $q^{2}$ ). However the transverse spatial components $\boldsymbol{q}^{\perp}$ of $q^{\mu}$ vanish, by definition. So this complication does not arise for the transverse $\mathrm{R}$ current operator $j^{\perp}$ that we discussed earlier, and (5.19) can also be used in that case.

So far, we have only looked at the operator $\mathcal{O}$ and not the other factors in the source term (5.16). We can rewrite (5.16) in $q$ space as

$$
\int_{q} \tilde{\Lambda}_{L}^{*}(q-\bar{k}) \mathcal{O}(q)
$$

Correspondingly attaching a factor of $\Lambda_{L}^{*}(q-\bar{k})$ to each $\mathcal{O}(q)$ in $(5.17)$, we get a probability distribution for $q$ proportional to

$$
\theta\left(q^{0}\right) \rho(q)\left|\tilde{\Lambda}_{L}(q-\bar{k})\right|^{2} \propto \theta\left(-q^{2}\right)\left(-q^{2}\right)^{\nu}\left|\tilde{\Lambda}_{L}(q-\bar{k})\right|^{2} .
$$

For a transverse-translation invariant source, the relative probability distribution for creating a jet with a given $q_{+}$, where $q^{2} \simeq 4 E q_{+}$, is then

$$
\mathcal{P}_{+}\left(\bar{q}_{+}\right)=\frac{\theta\left(-\bar{q}_{+}\right)\left|\bar{q}_{+}\right|^{\nu} \int d \Delta q_{-}\left|\tilde{\Lambda}_{L}^{(2)}\left(\bar{q}_{+}, \Delta q_{-}\right)\right|^{2}}{\int d q_{+} d \Delta q_{-} \theta\left(-q_{+}\right)\left|q_{+}\right|^{\nu}\left|\tilde{\Lambda}_{L}^{(2)}\left(q_{+}, \Delta q_{-}\right)\right|^{2}},
$$

which generalizes (4.7). We will repackage this as

$$
\mathcal{P}_{+}\left(q_{+}\right)=\frac{2^{(3+\nu) / 2} L}{\Gamma\left(\frac{1+\nu}{2}\right)} \theta\left(-q_{+}\right)\left|q_{+} L\right|^{\nu} \Psi_{\nu}\left(q_{+} L\right),
$$

\footnotetext{
${ }^{10}$ Alternatively, we could use the finite-temperature relation $i G_{>}(q)=\left[1+n\left(q^{0}\right)\right] \rho(q)$, where $n(\omega)=$ $\left(e^{\beta \omega}-1\right)^{-1}$ is the Bose distribution, and then use the fact that $q^{0} \simeq E \gg T$ in our problem.

${ }^{11}$ To get the dimension of $\rho(q)$, use (5.17) and note that $\mathcal{O}(x)$ having dimension $\Delta$ means that the Fourier transform $\mathcal{O}(q)$ has dimension $\Delta-d$.
} 
where

$$
\Psi_{\nu}\left(\bar{q}_{+} L\right) \equiv \frac{\Gamma\left(\frac{1+\nu}{2}\right) \int d q_{-}\left|\tilde{\Lambda}_{L}^{(2)}\left(\bar{q}_{+}, q_{-}\right)\right|^{2}}{2^{(3+\nu) / 2} L \int d q_{+} d q_{-} \theta\left(-q_{+}\right)\left|q_{+} L\right|^{\nu}\left|\tilde{\Lambda}_{L}^{(2)}\left(q_{+}, q_{-}\right)\right|^{2}}
$$

has been normalized so that $\Psi_{\nu}(0)=1$ in the case of a Gaussian source envelope (1.5).

Following (4.11), the distribution of stopping distances is then

$$
\begin{aligned}
\operatorname{Prob}\left(x^{3}\right) & \simeq \int d \epsilon \mathcal{P}_{+}(-\epsilon) \delta\left(x^{3}-x_{\text {stop }}^{3}(\epsilon)\right), \\
& \simeq \frac{2^{(3+\nu) / 2} L}{\Gamma\left(\frac{1+\nu}{2}\right)} \int_{0}^{\infty} d \epsilon(\epsilon L)^{\nu} \Psi_{\nu}(-L \epsilon) \delta\left(x^{3}-\frac{c}{2}\left(\frac{E}{\epsilon}\right)^{1 / 4}\right) \\
& =\frac{16\left(\sqrt{2} c^{4} E L\right)^{1+\nu}}{\Gamma\left(\frac{1+\nu}{2}\right)\left(2 x^{3}\right)^{5+4 \nu}} \Psi_{\nu}\left(-\frac{c^{4} E L}{\left(2 x^{3}\right)^{4}}\right) .
\end{aligned}
$$

So the power-law tail in figure 1 has generalized to $\left(x^{3}\right)^{-(5+4 \nu)}=\left(x^{3}\right)^{3-4 \Delta}$, as shown in figure 4 . For the transverse $\mathrm{R}$ current operator, $\Delta=3$, which recovers our previous result of $\left(x^{3}\right)^{-9}$ in that case.

\subsection{Gaussian source envelope}

Throughout this paper, we have discussed two different scales $x_{\text {typical }}^{3} \sim(E L)^{1 / 4}$ and $x_{\max }^{3} \sim$ $E^{1 / 3}$ characterizing the stopping distance. In (5.15), we generalized the latter to $x_{\max }^{3} \sim$ $(E / \Delta)^{1 / 3}$ for the case of large $\Delta$. Now we will discuss the similar generalization of $x_{\text {typical }}^{3}$. In general, we will still have

$$
x_{\text {typical }}^{3} \sim\left(\frac{E}{\left(-q_{+}\right)_{\text {typical }}}\right)^{1 / 4}
$$

but the relation between the typical $q_{+}$of jets and the source envelope size $L$ for large $\Delta$ will depend on details of how the source envelope $\tilde{\Delta}_{L}(q)$ falls off for large $q_{+}$. That's because the probability distribution (5.22) for the $q_{+}$of the jet involves a competition between the $\left|\bar{q}_{+}\right|^{\nu}$ factor which favors large $\left|q_{+}\right|$and the $\left|\Lambda_{L}\left(\bar{q}_{+}, \Delta q_{-}\right)\right|^{2}$ factor which suppresses $\left|q_{+}\right| \gg L^{-1}$. The typical value of $q_{+}$represents a balance between the two and will scale with $\nu$. For the sake of a concrete example, we consider here the case of a Gaussian source envelope (1.5). In this case, the function $\Psi_{\nu}\left(q_{+} L\right)$ in (5.23) and (5.25) is simply

$$
\Psi_{\nu}\left(q_{+} L\right)=e^{-2\left(q_{+} L\right)^{2}} .
$$

The typical values of $q_{+}$from the probability distribution (5.23) then scale as

$$
\left(-q_{+}\right)_{\text {typical }} \sim \frac{\nu^{1 / 2}}{L} \sim \frac{\Delta^{1 / 2}}{L}
$$

corresponding to ${ }^{12}$

$$
x_{\text {typical }}^{3} \sim\left(\frac{E L}{\sqrt{\Delta}}\right)^{1 / 4} .
$$

\footnotetext{
${ }^{12}$ Alternatively, one could compute the average value of $x_{\text {stop }}^{3}$ directly from (5.25), giving $x_{\text {avg }}^{3}=$ $\frac{c \Gamma\left(\frac{3}{8}+\frac{\nu}{2}\right)}{2^{7 / 8} \Gamma\left(\frac{1}{2}+\frac{\nu}{2}\right)}(E L)^{1 / 4}$.
} 


\section{Conclusion}

The 5-dimensional particle picture provides a relatively easy way of understanding (from the gravity side of the calculation) the appearance of the scale $(E L)^{1 / 4}$ in jets created by finite-size sources in strongly coupled $\mathcal{N}=4$ super Yang Mills plasmas. By thinking about sources with different types of momentum distributions, such as figures $2 \mathrm{a}$ and $\mathrm{b}$, we have learned that it is the range of $q^{2}$ of the source which determines the range of stopping distances. Making $\left|q^{2}\right|$ larger causes the jet to stop sooner. For any finite size $L$ of source, the uncertainty principle implies that there will be a spread in the components of $q$ of at least order $1 / L$ and so a spread in $q^{2}$ of at least order $E / L$. As a result, almost all of the jets produced will travel distances $\lesssim(E L)^{1 / 4}$ [of order $(E L)^{1 / 4}$ in the case of figure 2 a and $\ll$ $(E L)^{1 / 4}$ in the case of figure 2b]. Events where a jet travels further (up to $E^{1 / 3}$ ) will always be rare if the source size $L$ is small compared to the maximum stopping distance scale $E^{1 / 3}$.

This interpretation, based on the 5-dimensional particle picture, provides an important clarification to our original derivation of the average distribution of charge deposition shown in figure 1. This average includes an average over all events. From the original result, it was unclear whether on not figure 1 qualitatively tracks how the jet deposits its energy, momentum, and so forth on an event-by-event basis. It might have been that every single jet produced deposits some of its energy at $x^{3} \sim E^{1 / 3}$ and most of its energy at $x^{3} \sim(E L)^{1 / 4}$. The success of the 5-dimensional particle interpretation, and in particular the success of (1.8), indicates that figure 1 instead reflects a probability distribution for how far the jet travels, and each individual jet dumps its energy and charge in a very localized region of $x^{3}$. It should be possible to independently verify this conclusion by calculating correlations of the charge deposition at different distances, which we will leave to future work.

Our analysis of massive 5-dimensional fields indicated that the maximum stopping distance $E^{1 / 3}$ depends on the type of operator used to create the jet and that the distance decreases as the conformal dimension of that operator increases. That is, the maximum stopping distance depends on the type of high-energy excitation created. This may shed some light on a discrepancy between (i) the stopping distances found here and in refs. [2, 4], which find $x_{\max }^{3} \sim E^{1 / 3}$, and (ii) those based on the evolution of classical strings in 5 dimensions [1, 3], which find the parametrically smaller result $x_{\max }^{3} \sim(E / \sqrt{\lambda})^{1 / 3}$, where $\lambda \equiv N_{\mathrm{c}} g^{2}$ is the large 't Hooft coupling. The gauge theory states corresponding to classical strings in the gravity dual may simply be states that are much more easily stopped by the strongly-coupled quark-gluon plasma than are the states created by the source operators considered in this paper. One could then ponder which (if either) might be more instructive for lessons about the theory of real QCD plasmas. In order to further clarify the differences between the two approaches, it would be interesting to find a 4-dimensional gauge-theory description of a source that could be precisely linked through duality to the 5 -dimensional initial classical string configurations that have been used to study jet quenching.

\section{Acknowledgments}

We thank Djordje Minic for suggesting that we examine the dependence of jet stopping distances on dimension $d$. We also thank Andreas Karsch for useful conversations. This 
work was supported, in part, by the U.S. Department of Energy under Grant No. DEFG02-97ER41027 and by a Jeffress research grant, GF12334.

\section{A Stopping distances in different dimensions}

The only relevant difference between $\mathrm{AdS}_{5}$-Schwarzschild space and $\mathrm{AdS}_{d+1^{-}}$-Schwarzschild space is that the blackening function $f=1-u^{2}$ in the metric (2.2) is replaced by $f=$ $1-u^{d / 2}[14]$. The stopping distance (2.3) then becomes

$$
\begin{aligned}
x_{\text {stop }}^{3} & =\int_{0}^{1} \frac{d u}{\sqrt{u\left(u^{d / 2}-\frac{q^{2}}{|\boldsymbol{q}|^{2}}\right)}} \simeq \int_{0}^{\infty} \frac{d u}{\sqrt{u\left(u^{d / 2}-\frac{q^{2}}{|\boldsymbol{q}|^{2}}\right)}} \\
& =\frac{c_{d}}{\sqrt{2}}\left(\frac{|\boldsymbol{q}|^{2}}{q^{2}}\right)^{(d-2) / 2 d} \simeq \frac{c_{d}}{\sqrt{2}}\left(\frac{E}{4 \epsilon}\right)^{(d-2) / 2 d},
\end{aligned}
$$

where $c_{d}=\sqrt{2} B\left(1+\frac{1}{d}, \frac{1}{2}-\frac{1}{d}\right), B$ is the Beta function, and we have assumed $d>2$. Taking $\epsilon \sim 1 / L$, the dominant stopping distance (1.9) for our original source of figure 2a then generalizes from $(E L)^{1 / 4}$ to

$$
x_{\text {typical }}^{3} \sim(E L)^{(d-2) / 2 d} .
$$

The quickest way to estimate the maximum stopping distance, generalizing $E^{1 / 3}$, is to estimate when the stopping distance (A.2) becomes as large as the source itself, as we did for $d=4$ in section 1 . The result is

$$
x_{\max }^{3} \sim E^{(d-2) /(d+2)} .
$$

The last result can also be obtained from a wave analysis by analyzing the poles of the retarded bulk-to-boundary propagator, just as was done for $d=4$ in ref. [4]. The scale of the exponential decay in $(1.1 \mathrm{~b})$ was determined by the imaginary part of the propagator pole closest to the real axis. Here we simply follow section 4.6.2 of ref. [4], generalizing to arbitrary $d$. The massless field equation for $A_{\perp}$ is

$$
\left[\partial_{u}^{2}-\frac{4 E q_{+}-u^{d / 2} E^{2}}{u}\right] A_{\perp}=0 .
$$

Changing variables to $U \equiv e^{-i 2 \pi /(d+2)} E^{4 /(d+2)} u$, the field equation becomes

$$
\left[-\partial_{U}^{2}+\left(U^{(d-2) / 2}-\frac{a}{U}\right)\right] A_{\perp}=0
$$

where

$$
a \equiv 4 E^{(d-2) /(d+2)} e^{-i \pi d /(d+2)} q_{+} .
$$

Poles of the bulk-to-boundary propagator occur when the Schrödinger-like equation (A.5) has a zero-energy bound state that vanishes at the origin. The smallest value of $a$ for which this occurs is $O(1)$, from which (A.6) gives that the pole closest to the origin has

$$
\operatorname{Im} q_{+}^{\text {pole }} \sim E^{-(d-2) /(d+2)} .
$$


So the response to the source falls exponentially as

$$
\left|e^{i q_{+}^{\text {pole }} x^{+}}\right|^{2} \sim e^{-\kappa_{d} E^{-(d-2) /(d+2)} x^{+}}
$$

for large $x^{+}$, for some constant $\kappa_{d}$. This behavior is consistent with (A.3).

\section{B Null geodesic in $\mathrm{AdS}_{5}-\mathrm{Schwarzschild}$}

For the sake of keeping this paper self-contained, we give here a brief derivation of (2.1). A null geodesic has ${ }^{13}$

$$
0=(d s)^{2}=d x^{\mu} g_{\mu \nu} d x^{\nu}+d x^{5} g_{55} d x^{5}
$$

and so

$$
\frac{d x^{5}}{d \lambda}=\frac{1}{\sqrt{g_{55}}}\left[-g_{\mu \nu} \frac{d x^{\mu}}{d \lambda} \frac{d x^{\nu}}{d \lambda}\right]^{1 / 2},
$$

where we will take $\lambda$ to be any affine parameter for the trajectory. Because of 4-dimensional translation invariance,

$$
g_{\mu \nu} \frac{d x^{\nu}}{d \lambda}
$$

is conserved and proportional to $q_{\mu}$, so that

$$
\frac{d x^{\mu}}{d \lambda} \propto g^{\mu \nu} q_{\nu}
$$

Dividing this equation by (B.2) gives

$$
\frac{d x^{\mu}}{d x^{5}}=\sqrt{g_{55}} \frac{g^{\mu \nu} q_{\nu}}{\left(-q_{\alpha} g^{\alpha \beta} q_{\beta}\right)^{1 / 2}},
$$

which in turn gives (2.1).

\section{More on the geometric optics approximation}

Here, we will go into a little more detail about the conditions for the geometric optics approximation, for space-time backgrounds with 4-dimensional translation invariance. For the sake of concreteness, we will consider the case of the source (4.1) used in our earlier work, where the source operator is a transverse-polarized $\mathrm{R}$ current and so is dual to a 5-dimensional transverse vector field $A_{\perp}=\bar{\varepsilon}_{\mu} A^{\mu}$ in the gravity description. The equation of motion for $A_{\perp}\left(q, x^{5}\right)$, where $q$ is the 4-momentum, has the form

$$
\frac{1}{\sqrt{-g}} \partial_{5}\left(\sqrt{-g} g^{\perp \perp} g^{55} \partial_{5} A_{\perp}\right)=g^{\perp \perp} q_{\mu} g^{\mu \nu} q_{\nu} A_{\perp}
$$

where $g^{\perp \perp}$ is the component of the inverse metric in the direction of the polarization, e.g. $g^{\perp \perp}=g^{11}=g^{22}$ for $\boldsymbol{q}$ in the $x^{3}$ direction. ${ }^{14}$ Now switch to coordinate

$$
\ell \equiv \int \sqrt{g_{55}} d x^{5}
$$

\footnotetext{
${ }^{13}$ This analysis applies whenever the background metric has 4-dimensional translation invariance and the $x^{5}$ coordinate is chosen so that $g_{5 \mu}$ vanishes.

${ }^{14}$ Here we are implicitly assuming rotational invariance of the background in the transverse plane.
} 
which parametrizes proper length in the direction of the fifth dimension, and note for future reference that

$$
\partial_{\ell}=\frac{1}{\sqrt{g_{55}}} \partial_{5}
$$

The equation of motion is then

$$
w^{-1} \partial_{\ell}\left(w \partial_{\ell} A_{\perp}\right)=q_{\mu} g^{\mu \nu} q_{\nu} A_{\perp},
$$

where

$$
w=\sqrt{-g_{(4)}} g^{\perp \perp}
$$

and $g_{(4)}=g^{55} g$ is the determinant of the 4-dimensional part of the 5-dimensional metric. (As another simple example, one could consider the case of a source operator dual to a massless scalar field, which would correspond instead to taking $w=\sqrt{-g_{(4)}}$ in the equation of motion.) Now define

$$
a \equiv \sqrt{w} A_{\perp}
$$

to get

$$
\partial_{\ell}^{2} a=\left[q_{\mu} g^{\mu \nu} q_{\nu}+\frac{1}{2 \sqrt{w}} \partial_{\ell}\left(\frac{\partial_{\ell} w}{\sqrt{w}}\right)\right] a .
$$

This looks just like a one-dimensional quantum mechanics problem in $\ell$ with wavenumber $k(\ell) \equiv \sqrt{2 m(E-V(\ell))}$ replaced by

$$
k(\ell)=\sqrt{-q_{\mu} g^{\mu \nu} q_{\nu}-\frac{1}{2 \sqrt{w}} \partial_{\ell}\left(\frac{\partial_{\ell} w}{\sqrt{w}}\right)} .
$$

The WKB condition that the wavelength $\lambda(\ell)=2 \pi / k(\ell)$ in such a quantum mechanics problem not change significantly over distances of one wavelength is $\partial_{\ell} \lambda(\ell) \ll 1$.

For the metric (2.2), $w=(R / 2)^{2} f^{1 / 2} u^{-1}$, and (C.8) becomes

$$
k(u)=\frac{2}{R} \sqrt{\frac{u}{f}\left(u^{2} \boldsymbol{q}^{2}-q^{2}\right)+\frac{1-2 u^{2}}{4 f}}
$$

with the help of (C.3). For the $u$ 's of interest to our discussion of particles, which are $u \sim u_{\star} \ll 1$, the $\left(1-2 u^{2}\right) / 4 f$ term under the square root is negligible, giving

$$
k(u) \simeq \sqrt{-q_{\mu} g^{\mu \nu} q_{\nu}} \simeq \frac{2}{R} \sqrt{u\left(u^{2} \boldsymbol{q}^{2}-q^{2}\right)} .
$$

Again using (C.3), the condition $\partial_{\ell} \lambda(\ell)=\partial_{\ell}(2 \pi / k) \ll 1$ then gives (3.6) for $u \sim u_{\star}$.

\section{Maximum stopping distance for high-dimension source operators}

In section 5.1, we saw that the particle picture breaks down when $x^{3} \gtrsim x_{\max }^{3}$ with

$$
x_{\max }^{3} \sim\left(\frac{E}{\max (1, R m)}\right)^{1 / 3} \sim\left(\frac{E}{\Delta}\right)^{1 / 3}
$$


and we suggested that this $x_{\max }^{3}$ was the furthest that jets would propagate - that is, that energy or charge deposition at larger distances would be exponentially suppressed, similar to our previous $\Delta=3$ result of (1.1b). In this appendix, we will discuss how (D.1) arises in a wave analysis following the methods of ref. [4]. We will focus on the case of $\Delta \gg 1$. As in the main text, we assume that the large $E$ limit is taken first, and only then do we consider large $\Delta$.

There is a subtlety to the results we will find. In section D.2, we will analyze the exponential fall-off of jet charge deposition at very large $x$ by finding the location in the complex $q_{+}$plane corresponding to the first quasi-normal mode of the bulk field. For large $\Delta$, we will find an exponential fall-off of the form

$$
\exp \left(-\frac{2 c_{1}^{\prime} x^{3}}{E^{1 / 3} / \Delta^{4 / 3}}\right)
$$

where $c_{1}^{\prime}$ is a constant. This is the large- $\Delta$ version of the exponential in (1.1b). The $x^{3}$ scale that determines the rate of exponential fall-off in (D.2) is $E^{1 / 3} / \Delta^{4 / 3}$. One might naturally guess that exponential suppression therefore applies whenever $x^{3} \gg E^{1 / 3} / \Delta^{4 / 3}$ and so guess that $x_{\max }^{3} \sim E^{1 / 3} / \Delta^{4 / 3}$ instead of (D.1). This guess fails, however, for reasons we shall now outline.

\section{D.1 Overview}

To understand the issues involved, we briefly highlight some relevant aspects of the $m=0$ calculation from ref. [4]. The main part of the calculation there involved computing the bulk response $\mathcal{A}$ to the high-energy source on the boundary, given by

$$
\mathcal{A}(x, u) \equiv \int_{q} \mathcal{G}^{\mathrm{R}}(q, u) \tilde{\Lambda}_{L}(q-\bar{k}) e^{i q \cdot x}
$$

where $\mathcal{G}$ is the bulk-to-boundary propagator. Our result for the stopping distance came from extracting the behavior of $\mathcal{A}$ near the horizon, $u \rightarrow 1 .^{15}$ The critical part of the $q$ integration was the integral over $q_{+}$. For $x^{3} \ll E^{1 / 3}$ (in the $m=0$ case), we found that we could deform the $q_{+}$integration contour in the complex plane so that the integral was everywhere exponentially suppressed except at a saddle point ${ }^{16}$

$$
q_{+}^{\star} \simeq-\frac{c^{4} E}{\left(2 x^{3}\right)^{4}}
$$

\footnotetext{
${ }^{15}$ Readers may wonder at the connection between (i) studying $u \rightarrow 1$ and (ii) studying $u \sim u_{\star} \ll 1$ as in the particle arguments in the main text of this paper. The point is that how far the particle travels is determined by where it is when $u \rightarrow 1$, but the integral which gives that distance is dominated by $u \sim u_{\star} \ll 1$. In the wave analysis of ref. [4], we studied the response at late times, corresponding to $u \rightarrow 1$ for the bulk excitation. Our results for the near-horizon bulk response were determined by the $E^{1 / 4}\left(-q_{+}\right)^{3 / 4}$ term in the WKB exponent $S$ for $\mathcal{G}$ (see eq. (4.51) of ref. [4]). But this term was generated by the $u \sim u_{\star}$ region of the integral that gave $S$. See, for example, eq. (D13) of ref. [4], which is proportional to the current paper's particle stopping distance integral (2.3).

${ }^{16}$ In ref. [4], we expressed formulas in terms of $X^{+} \equiv x^{+}-\tau(u)$ instead of $x^{3}$. As discussed in that paper, the late-time response is localized to $x^{-} \simeq-\tau(u)$ (see eq. (4.49) of ref. [4]), and so $X^{+} \simeq 2 x^{3}$.
} 


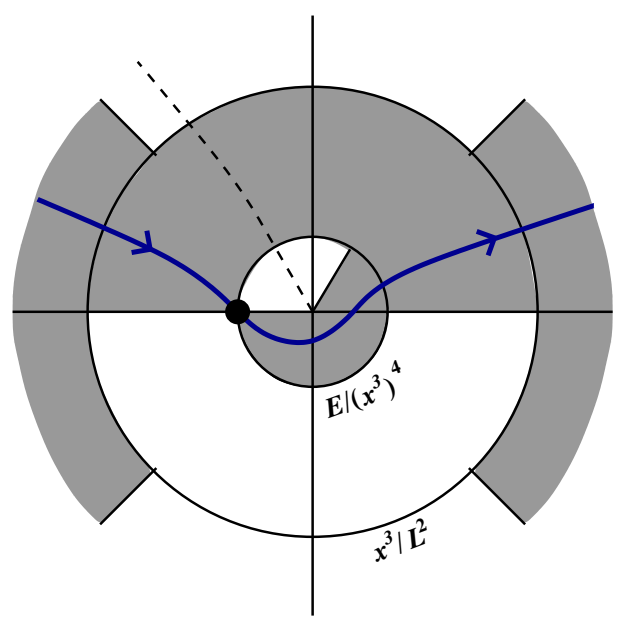

Figure 6. Integration contour in the $q_{+}$complex plane for saddle point approximations to the $q_{+}$ integral in (D.3). This is a slightly simplified version, appropriate for $u \rightarrow 1$, of figure 13b of ref. [4]. The location of the saddle point $q_{+}^{\star}$ is marked by the large dot. The circles indicate different parametric scales for $\left|q_{+}\right|$.

of (3.2). This contour is depicted in figure 6. Parametrically far into the interior of the shaded region indicates places where the magnitude of the integrand is exponentially suppressed. Parametrically far into the unshaded regions indicates places where it is exponentially large. The dashed line depicts a line of poles of the bulk-to-boundary propagator $\mathcal{G}^{\mathrm{R}}$, corresponding to quasi-normal modes. In the WKB approximation to that propagator, this line of poles became a cut.

For $x^{3} \gg E^{1 / 3}$, the regions of exponential suppression for the integrand are shown in figure 7a. Saddle point methods are unreliable. Instead, choose the integration contour shown there. The piece that goes around the line of poles can be re-expressed as a sum of contributions from each pole, as shown in figure $7 \mathrm{~b}$, which depicts a magnification of the neighborhood of the origin of figure 7a. The $e^{i q_{+} \cdot x^{+}}$piece of the $e^{i q \cdot x}$ in (D.3) causes the contributions from the poles to be exponentially suppressed according to their distance $\operatorname{Im} q_{+}$from the real axis. For $x^{3} \gg E^{1 / 3}$, the nearest pole dominates and produces the exponential fall-off (1.1b) of the jet's charge deposition.

Now we return to the massive case, with $\Delta \gg 1$, and ask what happens for

$$
\frac{E^{1 / 3}}{\Delta^{4 / 3}} \ll x^{3} \ll \frac{E^{1 / 3}}{\Delta^{1 / 3}} .
$$

This is the interesting case, where (i) the geometric optics and particle arguments of section 5 indicate that the charge deposition is not exponentially suppressed but (ii) the guess we might make based on (D.2) suggested that it is suppressed. As we shall discuss in section D.3, the mass does not significantly affect the massless picture of figure 6 provided

$$
\left|q_{+}\right| \gg \frac{\Delta^{4 / 3}}{E^{1 / 3}}
$$


(a)

(b)

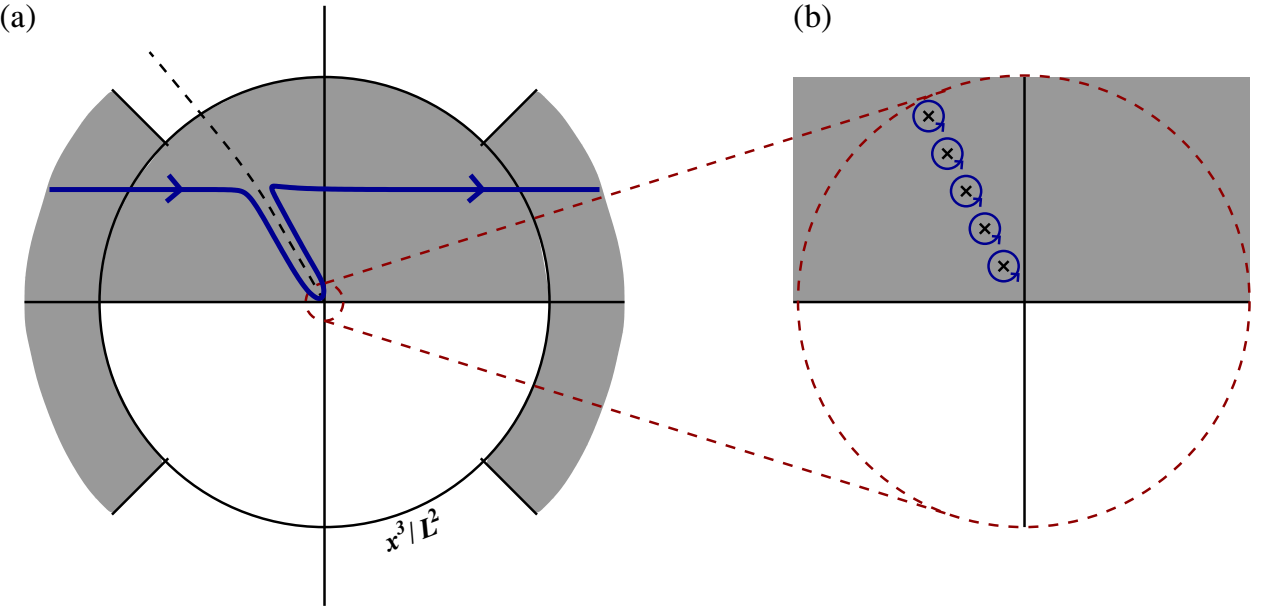

Figure 7. (a) Similar to figure 6 but for the case $x^{3} \gg x_{\max }^{3}$. (b) A magnification of the region near the origin.

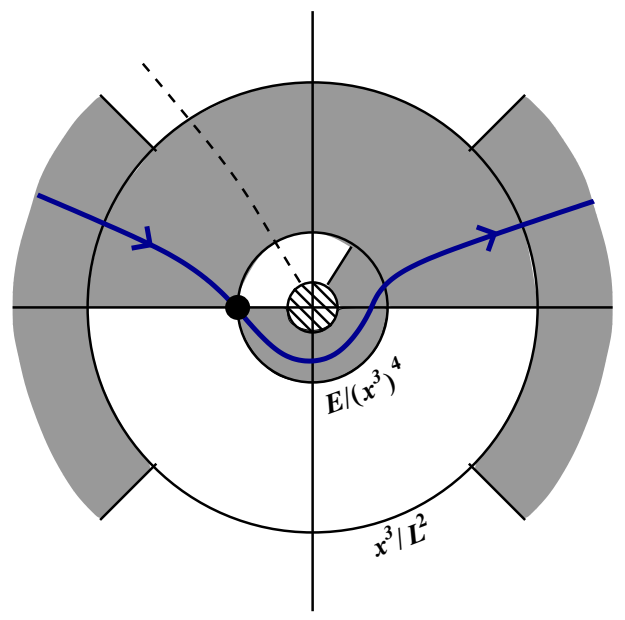

Figure 8. Like figure 6 but for the case of large $\Delta$ and $x^{3} \ll E^{1 / 3} / \Delta^{1 / 3}$. The cross-hatched region represents $\left|q_{+}\right| \lesssim \Delta^{4 / 3} / E^{1 / 3}$.

which is the condition (5.14) discussed in the main text. For $x^{3} \ll E^{1 / 3} / \Delta^{1 / 3}$ as in (D.5), the condition (D.6) is satisfied at the saddle point (D.4) and for larger $\left|q_{+}\right|$. So, as long as we are careful to route the contour as in figure 8, we can take over the methods of the $m=0$ calculation, make a saddle point approximation to the $q_{+}$integral (which corresponds to making the geometric optics approximation), and so find a result that is not exponentially suppressed.

But now consider a large $x^{3}$ calculation, along the lines of figure 7 . We shall see in section D.2 that the closest pole to the real axis has

$$
\operatorname{Im} q_{+}^{\text {pole }} \sim \frac{\Delta^{4 / 3}}{E^{1 / 3}}
$$


(a)

(b)

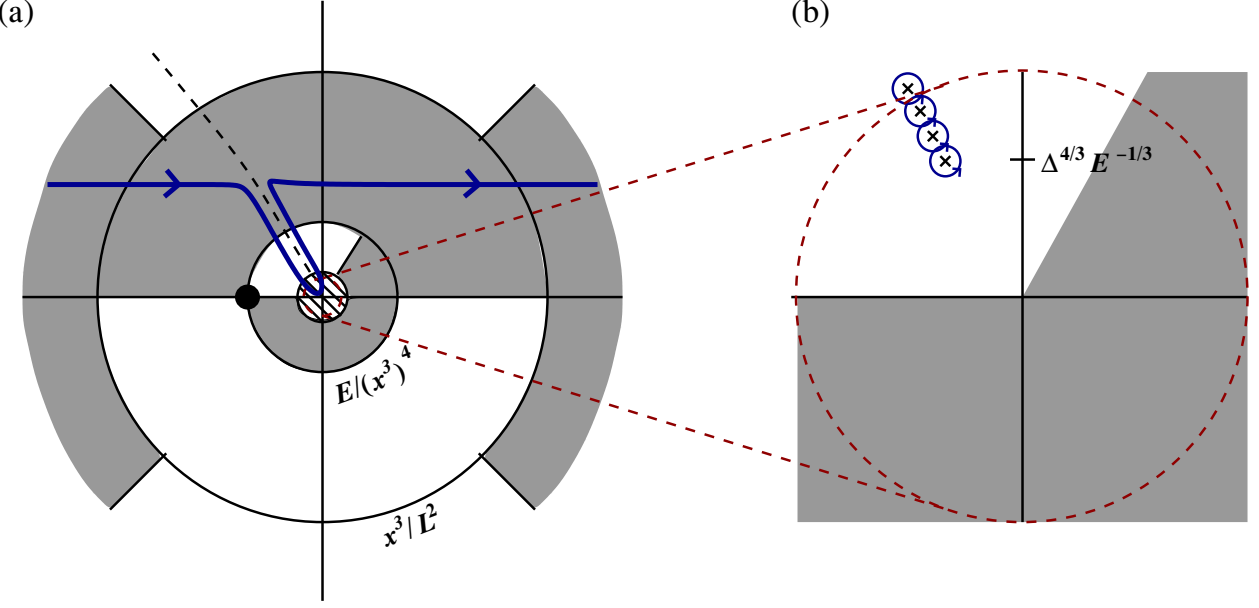

Figure 9. Like figure 8 but using a contour that picks up the poles instead of passing through the saddle point.

That is, the line of poles in figure 8 begins at the edge of the hatched circle. For $x^{3}$ in the range of (D.5), picking up the poles is not so useful. Figure 9 shows the large- $\Delta$ analog of figure 7 for this $x^{3}$ range. As one looks at poles progressively further from the origin, the $\exp (i q \cdot x)$ factors leads to suppression, as before, but the $\mathcal{G}^{\mathrm{R}}$ factor grows exponentially (as well as oscillates), and this exponential growth dominates the integrand in the unshaded region of figure 9 . So one cannot approximate the integral by the contribution from the nearest pole, and asymptotic formulas like (D.2) do not apply in this case.

Since the saddle point approximation is controlled and gives a result that is not exponentially suppressed in the range (D.5), whereas the approximations that lead to (D.2) are not valid there, we conclude that the maximum stopping distance is of order $E^{1 / 3} / \Delta^{1 / 3}$ and not $E^{1 / 3} / \Delta^{4 / 3}$.

What happens as one continues to increase $x^{3}$ ? For $x^{3} \gg E^{1 / 3} / \Delta^{1 / 3}$, the saddle point approximation breaks down and figure 9 becomes figure 10. In this case, the calculation is dominated by the first pole and (D.2) applies. We will not attempt here to calculate the details of the transitional behavior at $x \sim x_{\max }^{3} \cdot{ }^{17}$

\section{D.2 Quasi-normal modes}

To find the poles of $\mathcal{G}^{\mathrm{R}}$ in the complex $q_{+}$plane for large $E$, we follow the method of ref. [4]. For the sake of simplicity, we will focus on the case of a massive bulk scalar field, whose equation of motion is (5.7). As discussed in ref. [4], the pole positions at high energy are determined (up to small corrections) by the nature of the equation of motion for $u \ll 1$. It will be more convenient to work with the variable $z=2 \sqrt{u}$ instead of $u$. Writing

$$
\phi=z^{(d-1) / 2} \psi
$$

\footnotetext{
${ }^{17}$ However, for the sake of showing that (D.2) is mathematically consistent with $x_{\max }^{3} \sim E^{1 / 3} / \Delta^{1 / 3}$, we point out that $\left\{\exp \left[\frac{2 c_{1}^{\prime}\left(x-x_{\max }^{3}\right)}{E^{1 / 3} \Delta^{-4 / 3}}\right]+1\right\}^{-1}$ is an example of a function that is unsuppressed for $x \ll x_{\max }^{3}$ but decays like (D.2) for $x \gg x_{\max }^{3}$.
} 
(a)

(b)

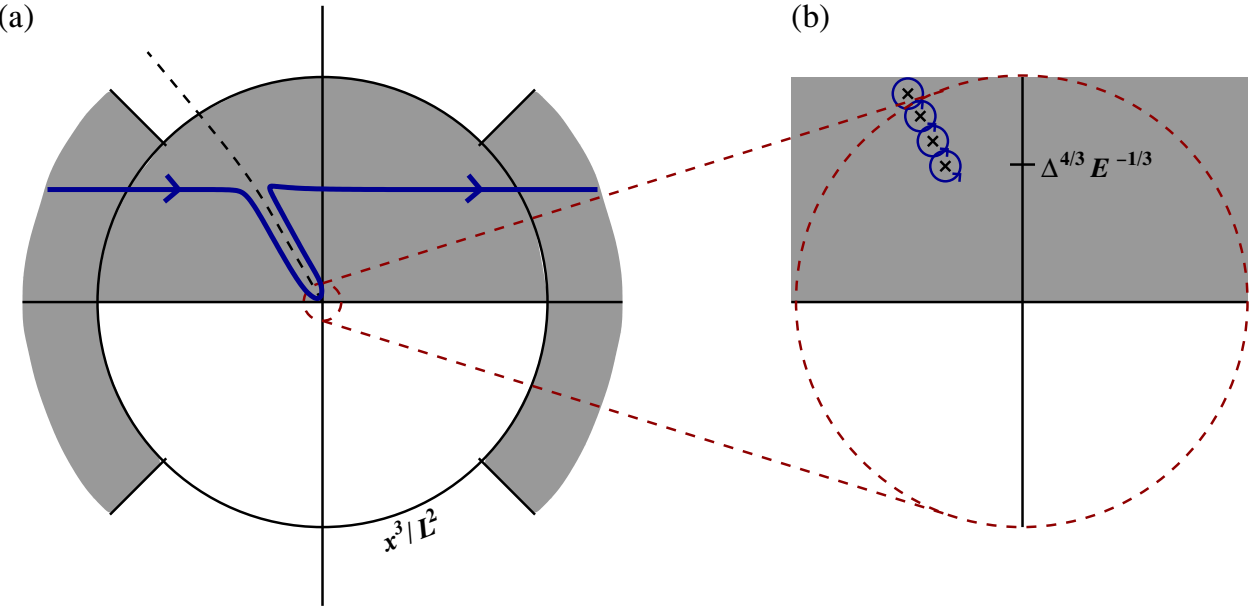

Figure 10. Like figure 9 but for the case $x^{3} \gg E^{1 / 3} / \Delta^{1 / 3}$.

the equation of motion for $u \ll 1$ becomes the Schrödinger-like equation

$$
-\frac{1}{2} \partial_{z}^{2} \psi+V(z) \psi=-\frac{1}{2} q^{2} \psi
$$

with potential

$$
V(z)=\frac{1}{2}\left[-\left(\frac{z}{2}\right)^{d} \boldsymbol{q}^{2}+\frac{(R m)^{2}+\frac{d^{2}-1}{4}}{z^{2}}\right] .
$$

Taking the high energy limit and and setting $d=4$, this is

$$
-\frac{1}{2} \partial_{z}^{2} \psi+V(z) \psi \simeq-2 E q_{+} \psi
$$

with

$$
V(z) \simeq \frac{1}{2}\left[-\left(\frac{z}{2}\right)^{4} E^{2}+\frac{\mathcal{M}^{2}}{z^{2}}\right]
$$

and

$$
\mathcal{M}^{2} \equiv(R m)^{2}+\frac{15}{4}
$$

Following ref. [4], make the change of variables from $u$ to

$$
U \equiv e^{-i \pi / 3} E^{2 / 3} u
$$

which turns the retarded boundary condition at large $u$ into the requirement that $\psi$ be real and exponentially falling. In terms of $z$, this redefinition is

$$
Z \equiv e^{-i \pi / 6} E^{1 / 3} z
$$

The resulting equation is ${ }^{18}$

$$
-\frac{1}{2} \partial_{Z}^{2} \psi+\mathcal{V}(Z) \psi=\frac{1}{2} a \psi
$$

\footnotetext{
${ }^{18}$ For comparison with ref. [4], one may write a similar equation in terms of $U$ by defining $\phi=u^{(d-2) / 4} \bar{\phi}$, giving $\left[-\partial_{U}^{2}+U+\frac{(R m)^{2}+3}{4 U^{2}}-\frac{a}{U}\right] \bar{\phi}=0$ for $d=4$. This reduces to (4.66) of ref. [4] for $A_{\perp}$ in the case
} 
with

$$
\mathcal{V}(Z)=\frac{1}{2}\left[\left(\frac{Z}{2}\right)^{4}+\frac{\mathcal{M}^{2}}{Z^{2}}\right]
$$

and $a$ defined in terms of $q_{+}$as in ref. [4]:

$$
q_{+}=\frac{1}{4} e^{i 2 \pi / 3} E^{-1 / 3} a .
$$

Solving (D.16) with the desired boundary conditions is equivalent to setting $a$ to be twice the bound-state energies associated with the potential $\mathcal{V}(z)$. For $\mathcal{M} \gg 1$, these can be well approximated by treating $\mathcal{V}(z)$ in harmonic-oscillator approximation around its minimum. The result is

$$
\frac{1}{2} a_{n}=\frac{3}{8} \mathcal{M}^{4 / 3}+\left(n+\frac{1}{2}\right) \sqrt{\frac{3}{2}} \mathcal{M}^{1 / 3}+O\left(\mathcal{M}^{-2 / 3}\right)
$$

for $n=0,1,2, \cdots .{ }^{19}$ Using (D.18), we find that the first pole in $q_{+}$is a distance of order $\Delta^{4 / 3} / E^{1 / 3}$ from the real axis, but the spacing between successive poles in figure 10b is only of order $\Delta^{1 / 3} / E^{1 / 3}$. The specific result for $a_{0}$ determines

$$
c_{1}^{\prime}=\frac{\sqrt{3} a_{0}}{4 \Delta^{4 / 3}} \simeq \frac{3 \sqrt{3}}{16}
$$

for the exponential fall-off (D.2) in the case $\Delta \gg 1$.

\section{D.3 Saddle point analysis}

In section D.1, we claimed that a large mass $m$ for the bulk field does not qualitatively change the massless saddle-point picture of figure 6 except inside the (avoidable) hatched region of figure 8 . Here, we will briefly sketch why. For $R m \gg 1$, the condition for the validity of the WKB approximation ${ }^{20}$ is satisfied in the small $u \rightarrow 0$ regime $u \ll u_{\text {min }}$ as well as in the oscillatory regime $u \gg u_{\min }$. (The turning point $u \sim u_{\min }$ can be avoided simply by analytically continuing around it, as in the textbook discussion of WKB in ref. [15]). So we may use WKB all the way to the boundary $u=u_{\mathrm{B}}$ :

$$
\mathcal{G}^{\mathrm{R}} \propto e^{i S} \equiv \exp \left[i \int_{u_{\mathrm{B}}}^{u} d u^{\prime} q_{5}\left(u^{\prime}\right)\right]
$$

where, for the sake of simplicity of presentation, we will suppress showing the WKB prefactor. For the massive case,

$$
q_{5}(u)=\frac{1}{f} \sqrt{\frac{u^{2}|\boldsymbol{q}|^{2}-q^{2}}{u}-\frac{(R m)^{2} f}{4 u^{2}}} .
$$

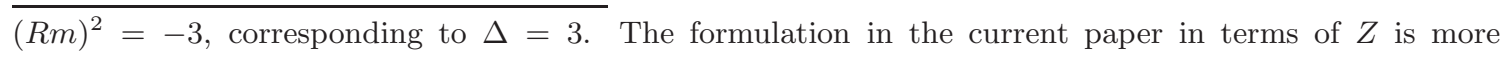
convenient because the pole locations $q_{+}$can be identified as proportional to the bound state energies of a Schrödinger potential $\mathcal{V}(Z)$. Note also that $\mathcal{M}^{2}$ plays a roll analogous to angular momentum squared in the Schrödinger problem (D.16), with $\mathcal{M}^{2} / Z^{2}$ like a centrifugal potential and the large- $\mathcal{M}$ limit analogous to a large angular momentum limit.

${ }^{19}$ Here we label the first pole $a_{0}$. In ref. [4] we instead called it $a_{1}$.

${ }^{20}$ Essentially: that the derivative of the WKB exponent does not change significantly over one e-folding or oscillation. 
The integrand in (D.3) then has exponential dependence

$$
e^{i q \cdot x} \mathcal{G}^{\mathrm{R}} \propto e^{i \mathcal{S}} \equiv e^{i(q \cdot x+S)},
$$

and the saddle point of its integral is determined by

$$
0=\frac{\partial \mathcal{S}}{\partial q_{\mu}}=\frac{\partial}{\partial q_{\mu}}\left[q \cdot x+\int d x^{5} q_{5}\left(x^{5}\right)\right]
$$

which gives

$$
x^{\mu}=-\int d x^{5} \frac{\partial q_{5}}{\partial q_{\mu}}
$$

Together with (D.22), this simply reproduces the particle-based formula (5.3) for the stopping distance. Approximating $q_{-} \simeq E$ and solving for $q_{+}$in terms of $x^{3}$ will then give the saddle point $q_{+}^{\star}$ for the $q_{+}$integration in (D.3). We've already discussed the effect of the mass on the particle stopping formula (5.3) back in section 5.1. Tracing the discussion of section 5.1 backward, $x^{3} \ll(E / \Delta)^{1 / 3}$ corresponds to $-q_{+}$given by $\epsilon \gg\left(E / \Delta^{4}\right)^{-1 / 3}$, which corresponds in turn to $u_{\min } \ll u_{\star}$. That's precisely the case where the mass had a negligible effect on the relationship between $x^{3}$ and $q_{+}$. In consequence, the mass $m$ will not have a significant effect on the determination of the saddle point $q_{+}^{*}$ for $x^{3} \ll(E / \Delta)^{1 / 3}$.

What about the behavior of the integrand elsewhere along the contour in figure 6 ? A discussion of the WKB exponent $S$ of (D.21) is complicated by the divergence (5.10) of the bulk-to-boundary propagator on the boundary, which shows up as a logarithmic divergence $\left(\propto \ln u_{\mathrm{B}}\right)$ of the integral in (D.21). We will briefly indicate in section D.4 how one can do a WKB analysis that avoids this divergence, but such details lose the forest for the trees. More simply, the $\ln u_{\mathrm{B}}$ divergence of $\int d u q_{5}$ is independent of $q_{+}$and so does not affect the $q_{+}$dependence of the integrand in (D.3), and so it will only affect the result by overall factors. To focus on the question of whether the mass makes a significant effect on the $q_{+}$ dependence, look at the effect of the mass on $\partial \mathcal{S} / \partial q_{+}$instead of on $\mathcal{S}$. So look at

$$
\frac{\partial \mathcal{S}}{\partial q_{\mu}}=x^{\mu}+\int d x^{5} \frac{\partial q_{5}}{\partial q_{\mu}} .
$$

The first term is mass independent, and the second term is just once again our integral for the particle stopping distance as a function of $q_{+}$, given by the right-hand side of (5.3), though with an imaginary part even for real negative $q_{+}$due to integrating over $u<$ $u_{\text {min }}$. However, if $\left|q_{+}\right| \gg \Delta^{4 / 3} / E^{1 / 3}$, then the effect of the mass on this integral will have negligible relative magnitude, just as in the previous discussion concerning the location of the saddle point.

\section{D.4 Avoiding WKB exponent divergences}

Finally, we sketch how one could set up a finite WKB integral if one wanted to carry through the analysis of this appendix in more detail than we have given. To get the normalization (5.10) appropriate for the bulk-to-boundary propagator $\mathcal{G}$, we want $\Phi$ to give $u_{\mathrm{B}}^{(d-\Delta) / 2}$ at $u=u_{\mathrm{B}}$. So

$$
\mathcal{G}^{\mathrm{R}} \approx u_{\mathrm{B}}^{(d-\Delta) / 2} \exp \left[i \int_{u_{\mathrm{B}}}^{u} d u^{\prime} q_{5}\left(u^{\prime}\right)\right]
$$


where we have again suppressed showing the WKB prefactor, other than the overall power of $u_{\mathrm{B}}$. We can trade the divergent $u_{\mathrm{B}}^{(d-\Delta) / 2}$ normalization factor for a finite $u^{(d-\Delta) / 2}$ by introducing a compensating change in the exponent:

$$
\mathcal{G}^{\mathrm{R}} \approx u^{(d-\Delta) / 2} \exp \left[i \int_{u_{\mathrm{B}}}^{u} d u^{\prime}\left(q_{5}\left(u^{\prime}\right)-i \frac{(\Delta-d)}{2 u^{\prime}}\right)\right] .
$$

In the large $\Delta$ limit (required for our WKB analysis in the region $u \ll u_{\min }$ ), $\Delta-d \simeq R m$, and so we will replace the last equation by ${ }^{21}$

$$
\mathcal{G}^{\mathrm{R}} \approx u^{(d-\Delta) / 2} \exp \left[i \int_{u_{\mathrm{B}}}^{u} d u^{\prime}\left\{\frac{1}{f\left(u^{\prime}\right)} \sqrt{\frac{u^{\prime 2}|\boldsymbol{q}|^{2}-q^{2}}{u^{\prime}}-\frac{(R m)^{2} f\left(u^{\prime}\right)}{4 u^{\prime 2}}}-i \frac{R m}{2 u^{\prime}}\right\}\right] .
$$

Now the integral in the exponent is finite if we take the limit $u_{\mathrm{B}} \rightarrow 0$, and so the appropriate WKB expression (still suppressing showing the original WKB prefactor) can be approximated as

$$
\mathcal{G}^{\mathrm{R}} \approx u^{(d-\Delta) / 2} \exp \left[i \int_{0}^{u} d u^{\prime}\left\{\frac{1}{f\left(u^{\prime}\right)} \sqrt{\frac{u^{\prime 2}|\boldsymbol{q}|^{2}-q^{2}}{u^{\prime}}-\frac{(R m)^{2} f\left(u^{\prime}\right)}{4 u^{\prime 2}}}-i \frac{R m}{2 u^{\prime}}\right\}\right] .
$$

One may then use this WKB formula to pursue a more detailed analysis. The $-i R m / 2 u^{\prime}$ subtraction in the integral cancels the original integrand for $u \ll u_{\min }$ and so keeps the integral finite. Its contribution for $u \gg u_{\min }$ will introduce an additive piece of approximately $\frac{i}{2} R m \ln u_{\min } \simeq \frac{i}{2} \Delta \ln u_{\min }$ in the WKB exponent $S$. That corresponds to a multiplicative factor of $u_{\min }^{-\Delta / 2}$ in the result for $e^{i \mathcal{S}}$ and so $u_{\min }^{-\Delta}$ in $|\mathcal{A}|^{2}$. Using (5.5) for $u_{\min }$ and then the saddle-point value (D.4) for $q_{+}$, this factor is

$$
u_{\min }^{-\Delta} \propto\left(q^{2}\right)^{\Delta} \propto\left(q_{+}^{\star}\right)^{\Delta} \propto\left(x^{3}\right)^{-4 \Delta},
$$

which is just the dependence of the power-law tail on $\nu \simeq \Delta$ that we previously found in $(5.25)$.

\section{References}

[1] S.S. Gubser, D.R. Gulotta, S.S. Pufu and F.D. Rocha, Gluon energy loss in the gauge-string duality, JHEP 10 (2008) 052 [arXiv:0803.1470] [SPIRES].

[2] Y. Hatta, E. Iancu and A.H. Mueller, Jet evolution in the $N=4$ SYM plasma at strong coupling, JHEP 05 (2008) 037 [arXiv:0803.2481] [SPIRES].

[3] P.M. Chesler, K. Jensen, A. Karch and L.G. Yaffe, Light quark energy loss in strongly-coupled $N=4$ supersymmetric Yang-Mills plasma, Phys. Rev. D 79 (2009) 125015 [arXiv:0810.1985] [SPIRES].

\footnotetext{
${ }^{21}$ We are sweeping something under the rug here. One makes a small relative error in replacing $\Delta-d$ by $R m$ when $\Delta$ is large, but in exponents one should really focus on absolute rather than relative errors. We leave further refinement to the interested reader.
} 
[4] P. Arnold and D. Vaman, Jet quenching in hot strongly coupled gauge theories revisited: 3-point correlators with gauge-gravity duality, JHEP 10 (2010) 099 [arXiv: 1008.4023] [SPIRES].

[5] S.S. Gubser and A. Yarom, Pointlike probes of superstring-theoretic superfluids, JHEP 03 (2010) 041 [arXiv:0908.1392] [SPIRES].

[6] P.M. Chesler, K. Jensen and A. Karch, Jets in strongly-coupled $N=4$ super Yang-Mills theory, Phys. Rev. D 79 (2009) 025021 [arXiv:0804.3110] [SPIRES].

[7] N. Itzhaki, J.M. Maldacena, J. Sonnenschein and S. Yankielowicz, Supergravity and the large- $N$ limit of theories with sixteen supercharges, Phys. Rev. D 58 (1998) 046004 [hep-th/9802042] [SPIRES].

[8] S. Lee, S. Minwalla, M. Rangamani and N. Seiberg, Three-point functions of chiral operators in $D=4, N=4 S Y M$ at large- $N$, Adv. Theor. Math. Phys. 2 (1998) 697 [hep-th/9806074] [SPIRES].

[9] E. Witten, Anti-de Sitter space and holography, Adv. Theor. Math. Phys. 2 (1998) 253 [hep-th/9802150] [SPIRES].

[10] D.Z. Freedman, S.D. Mathur, A. Matusis and L. Rastelli, Correlation functions in the CFT $(d) / A d S(d+1)$ correspondence, Nucl. Phys. B 546 (1999) 96 [hep-th/9804058] [SPIRES].

[11] S. de Haro, S.N. Solodukhin and K. Skenderis, Holographic reconstruction of spacetime and renormalization in the AdS/CFT correspondence, Commun. Math. Phys. 217 (2001) 595 [hep-th/0002230] [SPIRES].

[12] M. Bianchi, D.Z. Freedman and K. Skenderis, Holographic Renormalization, Nucl. Phys. B 631 (2002) 159 [hep-th/0112119] [SPIRES].

[13] K. Skenderis, Lecture notes on holographic renormalization, Class. Quant. Grav. 19 (2002) 5849 [hep-th/0209067] [SPIRES].

[14] E. Witten, Anti-de Sitter space, thermal phase transition and confinement in gauge theories, Adv. Theor. Math. Phys. 2 (1998) 505 [hep-th/9803131] [SPIRES].

[15] L. Landau and E. Lifshitz, Quantum Mechanics (Non-relativistic theory), 3rd edition Pergamon Press (1977). 